\title{
Ultrawide-Band Microwave Space-Time Beamforming for Hyperthermia Treatment of Breast Cancer: A Computational Feasibility Study
}

\author{
Mark Converse, Member, IEEE, Essex J. Bond, Student Member, IEEE, Susan C. Hagness, Member, IEEE, and \\ Barry D. Van Veen, Fellow, IEEE
}

\begin{abstract}
A new noninvasive ultrawide-band (UWB) microwave method for hyperthermia treatment of breast cancer is proposed. A train of UWB pulses are passed through a space-time beamformer and transmitted simultaneously from multiple antennas into the breast. The filters in the space-time beamformer are designed to compensate for dispersive propagation through breast tissue so that the pulses from each antenna add coherently at the treatment location and add incoherently elsewhere. Thus, the transmitted microwave energy is tightly focused at the treatment location to produce localized heating. The effectiveness of this procedure is shown by calculating the power density deposited in the breast using finite-difference time-domain (FDTD) electromagnetic simulations of realistic numerical breast phantoms derived from magnetic resonance images of patients. Both supine and prone patient configurations are considered. The robustness of our approach to variations in breast density and heterogeneity is demonstrated. We also present examples of temperature distributions calculated using the FDTD method applied to a simple thermal model for the breast phantoms. The results illustrate that, within the confines of this model, our UWB approach achieves sufficiently elevated temperatures in the vicinity of small tumors while maintaining safe temperatures throughout the remainder of the breast. The promising outcome of this feasibility study suggests that further development of this technique should be pursued.
\end{abstract}

Index Terms-Breast cancer, electromagnetic (EM) hyperthermia, finite-difference time-domain (FDTD) method, microwave imaging, space-time beamforming, ultrawide-band (UWB) radar.

\section{INTRODUCTION}

B REAST CANCER is one of the most common types of cancer and a major cause of death among women. Each year in the U.S., almost 180000 women are diagnosed with breast cancer. ${ }^{1}$ Treatment for early-stage breast cancer typically

Manuscript received October 18, 2003; revised March 17, 2004. This work was supported by the Department of Defense Breast Cancer Research Program under Award DAMD17-02-1-0625, by the National Science Foundation under Grant BES-0201880, and by the National Institutes of Health under Grant 1 F31 CA099933-01.

The authors are with the Department of Electrical and Computer Engineering, University of Wisconsin-Madison, Madison, WI 53706 USA (e-mail: converse@cae.wisc.edu; ejbond@wisc.edu; hagness@engr.wisc.edu; vanveen@engr.wisc.edu).

Digital Object Identifier 10.1109/TMTT.2004.832012

${ }^{1}$ National Cancer Institute, Bethesda, MD. [Online]. Available: http://www.nci.nih.gov/ involves a lumpectomy or partial mastectomy to remove the carcinoma and its margins, followed by radiation therapy to destroy any remaining cancer cells. For larger tumors, pre-operative chemotherapy may be used to shrink the tumor to conserve a larger portion of the breast.

Clinical studies have shown local hyperthermia to be effective in the treatment of breast cancer [1]-[3], as well as other types of cancer [4]-[7] when delivered as an adjuvant to radiation and/or chemotherapy. The objective of hyperthermia treatment is to raise the temperature in the tumor volume above 42 ${ }^{\circ} \mathrm{C}-43{ }^{\circ} \mathrm{C}$ for a sufficient period of time in order to achieve cell death or render the cells more sensitive to ionizing radiation and chemical toxins [8]. The persistent challenges with hyperthermia treatment are preferentially heating the cancerous tissue while maintaining the surrounding healthy tissue at temperatures well below $42{ }^{\circ} \mathrm{C}$ (i.e., avoiding the introduction of auxiliary foci, or "hot-spots") and noninvasively monitoring the thermal dose delivered to the cancerous tissue. Consequently, a great deal of research has been conducted to develop more effective techniques for hyperthermia treatment over the past two decades, primarily using RF, microwave, and ultrasound energy. The field of ultrasound hyperthermia has seen the most progress and is currently the most commonly used heating method. Both single-focused and phased-array transducers [9], [10] are employed to obtain preferential heating.

There have been a number of recent advances in electromagnetic (EM) hyperthermia techniques, applicator designs, and monitoring approaches [11]. Most of the investigations to date have focused on narrow-band (NB) techniques employing phased arrays. For example, Kowalski et al. [12] have proposed a method to selectively heat regions deep inside the body using EM annular phased arrays (APAs) with the aid of magnetic resonance imaging (MRI) for temperature estimation. A controller chooses the optimal amplitudes and phases of the RF driving signals of the APA based on a parametric model that relates the control inputs to the resultant temperature elevations. This approach can be incorporated in a closed-loop temperature feedback control system as demonstrated with simulations in [13]. The hyperthermia technique developed by Fenn et al. [14] employs an adaptive microwave phased-array technique to focus power at desired locations in the breast. Feedback is provided via invasive electric-field probes, which measure the EM energy deposited at various locations in the breast, including 
the tumor site. This system has recently been tested on rabbit thighs [15]. Jacobsen [16] recently proposed an alternative NB approach based on the use of multiple frequencies and an APA. Preliminary results suggest that distributing the transmitted power over a frequency band (in this case, three different sinusoidal components over a $520-\mathrm{MHz}$ band) produces fewer hot spots in the volume to be heated.

In this paper, we present a numerical investigation of the feasibility of using an ultrawide-band (UWB) microwave space-time beamforming system to focus microwave energy at a lesion site. We achieve this focus by first passing the UWB pulse train though a bank of finite-impulse response (FIR) filters, one in each antenna channel, which compensate for dispersive propagation effects. The signals are time delayed to compensate for the expected propagation delay of the signal traveling from the antenna to the focal spot and then simultaneously transmitted into the breast. This process creates constructive interference at the focal spot. An anatomically realistic two-dimensional (2-D) finite-difference time-domain (FDTD) EM breast model is used to simulate the absorbed EM power density distributions that result from our focusing algorithm. The effectiveness of the focusing algorithm is evaluated with respect to hypothesized variations in the breast tissue dielectric properties to demonstrate the robustness of this approach. Such variations are to be expected in a clinical setting due to the natural heterogeneity of breast tissue. A simple thermal model is also used to illustrate that the temperature requirements for hyperthermia treatment can be achieved by our UWB approach.

The proposed UWB microwave hyperthermia approach is a natural companion to the UWB space-time microwave imaging method of [17] for detecting and localizing lesions. First, the tumor is localized by sequentially transmitting low-power UWB pulses from antennas located near the surface of the breast. The received backscattered signals are passed through a space-time beamformer that is designed to image backscattered signal energy as a function of location. The low-power imaging scan of the breast inherently contains the information needed to tightly focus high power pulses at the detected site of the tumor. Thus, the transmit focusing algorithm described in this paper is used to launch high-power signals from all antennas simultaneously to focus energy in the breast. Our focusing algorithm enables the same antenna array to be used for the detection and treatment of lesions, leading to a noninvasive, cost-effective system. Furthermore, this coupling of the detection and treatment aspects facilitates the continuous updating of the precise location of the tumor site, thereby compensating for patient movement and introducing robustness to errors in assumed microwave properties of breast tissue. While the monitoring of thermal dose throughout the breast region is beyond the scope of this paper, we note that our approach is compatible with noninvasive temperature monitoring schemes currently under investigation, e.g., microwave [18]-[20] and ultrasound [21] thermometry.

The following section presents the beamformer design procedure used for focusing signals. In Section III, we describe the methods used to evaluate the feasibility of our focusing strategy. EM power density and temperature distributions are presented in Section IV to demonstrate the performance, followed by con- cluding remarks in Section V. Lower and upper case boldface Roman type are used to denote vector and matrix quantities, respectively. Superscripts $T$ and $H$ denote matrix transpose and Hermitian (complex-conjugate transpose), respectively, while superscript $*$ denotes complex conjugation.

\section{MicROWAVE HYPERTHERMIA VIA SPACE-TIME BEAMFORMING}

The use of a multiantenna array offers the opportunity for transmitting signals that constructively interfere at a desired location and, thus, provide selective heating. Constructive interference is obtained with NB (single-frequency) focusing methods [12], [14] by adjusting the amplitude and phase of a sinusoidal signal in each antenna channel to compensate for the expected radial spreading and time delay incurred when the signal propagates from the antenna to the target focal point. Our UWB approach uses a space-time beamformer to implement frequency-dependent amplitude and phase adjustments in each channel. Thus, analogous to NB methods, UWB methods also exploit constructive/destructive interference in space. However, an UWB approach further exploits incoherent combining of power across frequency and space. The mainlobe peak for each frequency component of the UWB signals occurs at the focal location, thus, the total power deposited at the focal location is equal to the integral over frequency of the power deposited by each frequency component. Away from the focal location, some frequencies have sidelobe peaks, while others have nulls so the combined power is proportionally less than the combined power at the focal location. Hence, UWB focusing may offer the potential for lower overall sidelobe levels than NB focusing, for a fixed mainlobe power. UWB focusing may also be more robust to variability in breast density between patients since the optimum frequency for focusing NB signals is dependent on the dielectric properties of the breast, which varies with breast density. Use of UWB signals for focusing eliminates this problem.

In order to achieve preferential heating, we design the space-time beamformer to achieve unit gain at the focus location $\mathbf{r}_{0}$, while minimizing gain elsewhere in the breast. The focus location $\mathbf{r}_{0}$ represents the location of the tumor, which can be determined using any number of different imaging techniques. However, there is an advantage to using the same propagation model for tumor localization as is used for transmit-focusing beamformer design. If mismatch between the assumed propagation model and actual propagation characteristics introduces an error in estimated tumor location, the mismatch resulting from the use of the same propagation model for transmit focusing will cause energy to be focused at the true tumor location.

Fig. 1 depicts a block diagram of a time-domain beamformer implementation for depositing energy at $\mathbf{r}_{0}$. In this paper, we assume the antenna array contains $N=17$ elements. Furthermore, the UWB microwave signal $i[n]$ is assumed to be a discrete differentiated Gaussian with full-width at half maximum equal to $110 \mathrm{ps}$ for a sampling interval of $T_{s}=20 \mathrm{ps}$. The bandwidth of this pulse approximately extends from 1 to $11 \mathrm{GHz}$ 


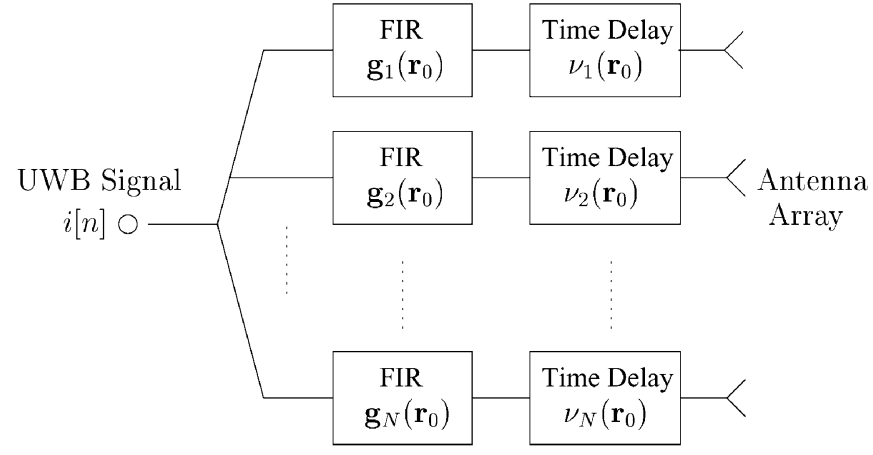

Fig. 1. Block diagram of the space-time beamformer used for depositing energy at location $\mathbf{r}_{0}$.

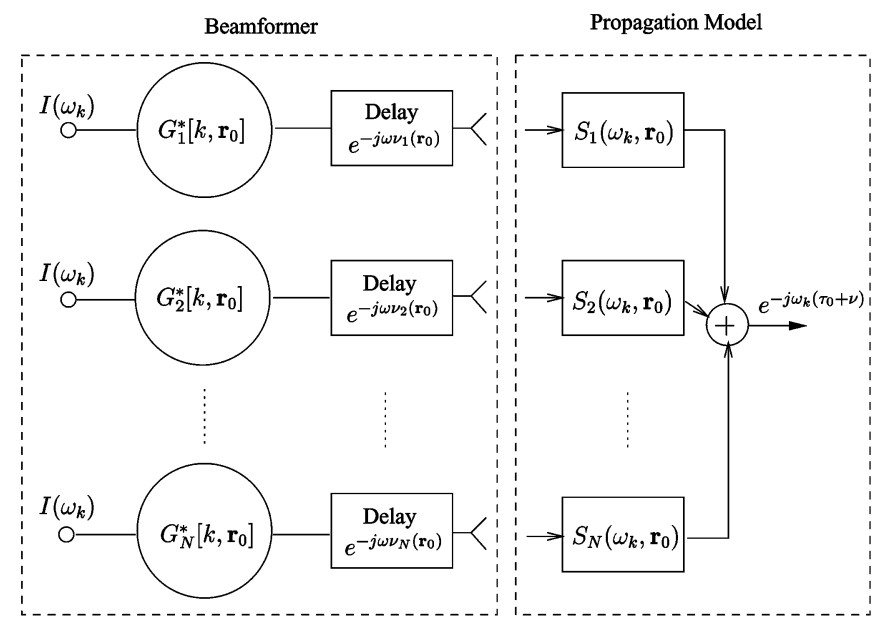

Fig. 2. Block diagram of the frequency-domain beamforming implementation used to design FIR filters to focus energy at location $\mathbf{r}_{0}$

with peak spectral energy near $6 \mathrm{GHz}$. In each channel, $i[n]$ passes through a FIR filter that compensates for dispersive propagation effects [22]. The signal in each channel is then time delayed to compensate for bulk propagation delay. The time-delayed signals are simultaneously transmitted into the breast and coherently combine at location $\mathbf{r}_{0}$.

Although the UWB approach requires a time-domain implementation to simultaneously transmit UWB pulses, the design of the space-time beamformer can be performed in the time or frequency domain, just as with beamformer design for tumor detection [17], [23]. We choose the frequency-domain approach, as it requires substantially less computation than that required by the time-domain approach. A block diagram of a conceptual frequency-domain beamforming implementation employed for design is shown in Fig. 2. Here, $I\left(\omega_{k}\right)$ is the $M$-point discrete Fourier transform (DFT) of the UWB signal $i[n]$, where $\omega_{k}$ is the $k$ th DFT frequency. The DFT length $M$ is determined using the travel time to a point in the imaging region at maximum distance from the antennas. We choose $M=63$ points so $\omega_{k}$ spans dc to $50 \mathrm{GHz}$ in 63 uniform steps. At each frequency, the FIR filters in Fig. 1 are represented by multiplication by complex constants $G_{\ell}^{*}\left[k, \mathbf{r}_{0}\right], \ell=1,2, \ldots, N$ in Fig. 2 , and the time delay corresponds to a phase shift that is a linear function of frequency. Here, $\nu_{\ell}\left(\mathbf{r}_{0}\right)=\nu-\tau_{\ell}\left(\mathbf{r}_{0}\right), \ell=1,2, \ldots, N$, where the delay $\tau_{\ell}\left(\mathbf{r}_{0}\right)$ is computed from the average wave propagation speed and the distance between the $\ell$ th antenna and the focal point $\mathbf{r}_{0}$. We choose $\nu$ to be an arbitrary delay greater than $\max _{\ell} \tau_{\ell}\left(\mathbf{r}_{0}\right)$ to ensure causality. The model for propagation from the transmitter to location $\mathbf{r}_{0}$ is depicted on the right-hand side of this figure. The term $S_{\ell}\left(\omega_{k}, \mathbf{r}_{0}\right)$ is an analytical model of the frequency response associated with propagation from the $\ell$ th antenna to location $\mathbf{r}_{0}$.

Denote the beamformer weight in the $\ell$ th channel at DFT index $k$ as $G_{\ell}\left[k, \mathbf{r}_{0}\right]$. As in [23], the beamformer is designed only at the frequencies that lie in the UWB pulse band and set to zero at frequencies that lie outside of this band. In order for the beamformer to compensate for one-way propagation to location $\mathbf{r}_{0}$, we require the combined effects of the coarse time alignment and propagation to have unit gain and linear phase, as shown in Fig. 2, and is given by

$$
\sum_{\ell=1}^{N} G_{\ell}^{*}\left[k, \mathbf{r}_{0}\right] e^{-j \omega_{k} \nu_{\ell}\left(\mathbf{r}_{0}\right)} S_{\ell}\left(\omega_{k}, \mathbf{r}_{0}\right)=e^{-j \omega_{k}\left(\tau_{0}+\nu\right)} .
$$

The linear phase term on the left-hand side of (1), i.e., $e^{-j \omega_{k} \nu_{\ell}\left(\mathbf{r}_{0}\right)}$, represents the time delay in Fig. 2, and $\tau_{0}=T_{s}(M-1) / 2$ is the average time delay introduced by the length $M$ FIR filters with sampling interval $T_{s}$. Let $\tilde{S}_{\ell}\left(\omega_{k}, \mathbf{r}_{0}\right)$ be the frequency response remaining after factoring the linear phase-shift term associated with the one-way propagation delay out of $S_{\ell}\left(\omega_{k}, \mathbf{r}_{0}\right)$

$$
S_{\ell}\left(\omega_{k}, \mathbf{r}_{0}\right)=\tilde{S}_{\ell}\left(\omega_{k}, \mathbf{r}_{0}\right) e^{-j \omega_{k} \tau_{\ell}\left(\mathbf{r}_{0}\right)} .
$$

Equation (1) can be rewritten as

$$
\sum_{\ell=1}^{N} G_{\ell}^{*}\left[k, \mathbf{r}_{0}\right] \tilde{S}_{\ell}\left(\omega_{k}, \mathbf{r}_{0}\right)=e^{-j \omega_{k} \tau_{0}} .
$$

Now form the column vectors

$$
\begin{aligned}
& \mathbf{v}\left(\omega_{k}, \mathbf{r}_{0}\right)=\left[\tilde{S}_{1}\left[k, \mathbf{r}_{0}\right], \ldots, \tilde{S}_{N}\left[k, \mathbf{r}_{0}\right]\right]^{T} \\
& \mathbf{g}\left(\omega_{k}, \mathbf{r}_{0}\right)=\left[G_{1}\left[k, \mathbf{r}_{0}\right], \ldots, G_{N}\left[k, \mathbf{r}_{0}\right]\right]^{T} .
\end{aligned}
$$

This allows for (3) to be written compactly as

$$
\mathbf{g}^{H}\left(\omega_{k}, \mathbf{r}_{0}\right) \mathbf{v}\left(\omega_{k}, \mathbf{r}_{0}\right)=e^{-j \omega_{k} \tau_{0}} .
$$

We employ a penalized least squares solution to (4) that trades solving the constraints of (4) exactly against the robustness of the solution. The robustness of a beamformer to errors between actual and assumed propagation models and to background noise is proportional to the norm of the weight vector [24], [25] or noise gain. The penalized least-squares problem is formulated as

$$
\begin{array}{r}
\mathbf{g}\left(\omega_{k}, \mathbf{r}_{0}\right)=\arg \min _{\mathbf{g}}\left|\mathbf{g}^{H}\left(\omega_{k}, \mathbf{r}_{0}\right) \mathbf{v}\left(\omega_{k}, \mathbf{r}_{0}\right)-e^{-j \omega_{k} \tau_{0}}\right|^{2} \\
+\xi \mathbf{g}^{H}\left(\omega_{k}, \mathbf{r}_{0}\right) \Lambda\left[k, \mathbf{r}_{0}\right] \mathbf{g}\left(\omega_{k}, \mathbf{r}_{0}\right)
\end{array}
$$

where $\Lambda\left[k, \mathbf{r}_{0}\right]$ is a matrix representing the structure of the penalty, and $\xi$ is a real nonnegative parameter whose value is selected to scale the impact of the penalty. We set $\xi$ to 1 . The matrix $\Lambda\left[k, \mathbf{r}_{0}\right]$ is chosen to be a diagonal matrix with entries $\left\{\left|\tilde{S}_{1}\left[k, \mathbf{r}_{0}\right]\right|, \ldots,\left|\tilde{S}_{N}\left[k, \mathbf{r}_{0}\right]\right|\right\}$ as this choice offers a 
balance between white noise gain and the sensitivity to tissue propagation errors [26]. Using this choice for the penalty, the solution to (5) is obtained as

$$
G_{\ell}\left[k, \mathbf{r}_{0}\right]=\frac{\tilde{S}_{\ell}\left[k, \mathbf{r}_{0}\right] e^{j \omega_{k} \tau_{0}}}{\left|\tilde{S}_{\ell}\left[k, \mathbf{r}_{0}\right]\right|\left(\xi+\sum_{m=1}^{N}\left|\tilde{S}_{m}\left[k, \mathbf{r}_{0}\right]\right|\right)}
$$

The FIR filter weights in the $\ell$ th channel, i.e., $\mathbf{g}_{\ell}\left(\mathbf{r}_{0}\right)$, are obtained by performing the inverse DFT of $G_{\ell}\left[k, \mathbf{r}_{0}\right]$.

The propagation model used in the design of the beamformer assumes transmission through two uniform dielectric media: a coupling medium consisting of deionized water and normal breast tissue. Note that skin and malignant breast tissue media are not included in the propagation model. The antenna array is located in the coupling medium near the breast medium. An estimate of the minimum distance, i.e., $d_{c_{\ell}}$, the UWB pulse travels in the coupling medium from the $\ell$ th antenna to the breast surface, which is obtained by processing the received signals obtained from a low-power transmission of the UWB signals. The dominant backscattered response in each received signal is due to the interfaces between the coupling medium, skin, and breast. By matched filtering the transmitted signal with each received waveform, an estimate of the round-trip time delay from the transmitting antenna to the interface is obtained. Multiplying half of this time delay with the average wave propagation speed in the coupling medium yields the estimate of $d_{c_{\ell}}$. For any location $\mathbf{r}$ in the breast region, we assume that the distance the pulse travels in the breast, i.e., $d_{b_{\ell}}(\mathbf{r})$, is given by the distance from the $\ell$ th antenna to location $\mathbf{r}$ minus the estimated distance the UWB pulse travels in the coupling medium

$$
d_{b_{\ell}}(\mathbf{r})=\left|\mathbf{r}-\mathbf{r}_{\ell}\right|-d_{c_{\ell}}
$$

where $\mathbf{r}_{\ell}$ is the location of the $\ell$ th antenna.

The monostatic frequency response $S_{\ell}(\omega, \mathbf{r})$ incorporates the frequency-dependent propagation effects in the coupling medium and normal breast tissue, explicitly shown by the product of two quantities in the following equation:

$$
\begin{aligned}
S_{\ell}(\omega, \mathbf{r})=\left[\frac{1}{\sqrt{d_{c_{\ell}}}} e^{-\alpha_{c}(\omega) d_{c_{\ell}}} e^{-j \beta_{c}(\omega) d_{c_{\ell}}}\right] \\
\times\left[\frac{1}{\sqrt{d_{b_{\ell}}(\mathbf{r})}} e^{-\alpha_{b}(\omega) d_{b_{\ell}}(\mathbf{r})} e^{-j \beta_{b}(\omega) d_{b_{\ell}}(\mathbf{r})}\right]
\end{aligned}
$$

where $\alpha_{c}(\omega)$ and $\alpha_{b}(\omega)$ are the frequency-dependent attenuation constants in the coupling medium and breast, respectively, and $\beta_{c}(\omega)$ and $\beta_{b}(\omega)$ are the frequency-dependent phase constants in the coupling medium and breast, respectively. A farfield approximation is inherent in (8). Although this propagation model involves several simplifying assumptions, we show in Section IV that this model results in designs that are effective in realistic scenarios.

\section{Numerical Models for Performance Evaluation}

We investigate the feasibility of UWB space-time beamforming for hyperthermia treatment using 2-D simulations to

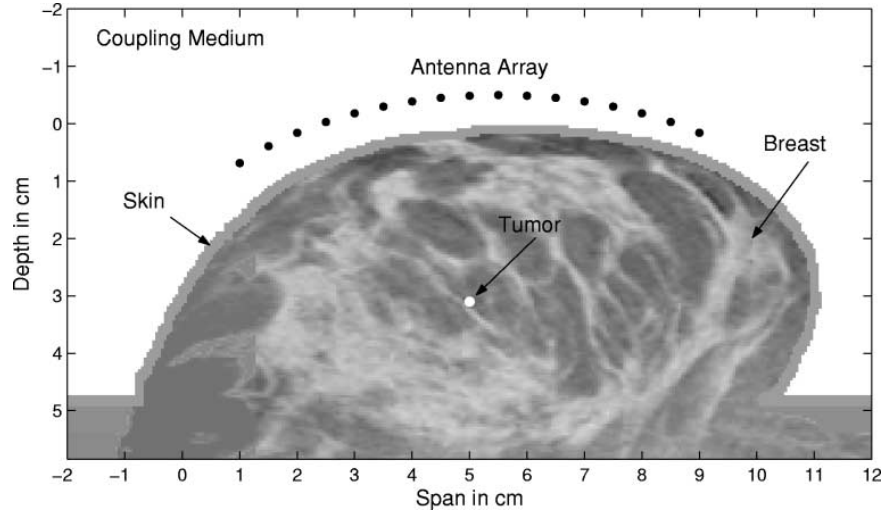

(a)

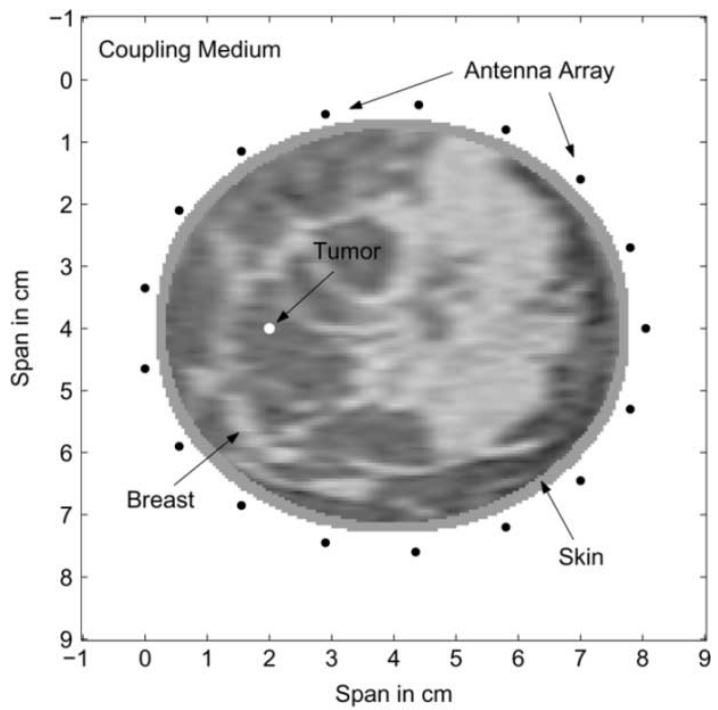

(b)

Fig. 3. 2-D MRI-derived FDTD breast model for a patient lying in the: (a) supine and (b) prone positions. Each model contains a 2-mm-diameter malignant lesion denoted by the white dot. The 17 black dots away from the surface of the breast represent antenna locations.

calculate distributions of absorbed power density and temperature throughout the breast. This approach allows for rapid evaluation of the potential performance of our proposed UWB hyperthermia technique. Our previous experience with successfully extending receive-focusing beamformer designs from 2-D [17], [23] to three-dimensional (3-D) [27] suggests that the 2-D design and implementation of the transmit-focusing beamformers presented here can also be readily extended to 3-D. We also expect that the performance in 3-D will be qualitatively similar to that presented here in 2-D.

\section{A. Anatomically Based Breast Phantoms}

Two possible patient positions are considered in this feasibility study. In both configurations, the breast is immersed in a deionized-water bolus, which not only serves as a coupling medium, but also provides superficial cooling. The first position involves the patient lying in the supine (or face-up) position. In this position, the antenna array is placed near the surface of the naturally flattened breast. This configuration allows better access to smaller breast volumes and tumors adjacent to the chest wall. The second configuration involves the patient lying in the 
TABLE I

DEBYE PARAMETERS FOR THE DISPERSIVE MATERIALS INCLUDED IN THE EM Model, AND THE RESUlting Dielectric PROPERTIES AT $6 \mathrm{GHz}$ (MID-SPECTRUM) [28], [30]

\begin{tabular}{c||c|c|c|c|c|c}
\hline \multicolumn{1}{c||}{ Media } & \multicolumn{6}{c}{ Electromagnetic Media Characteristics } \\
\cline { 2 - 7 } & $\epsilon_{\infty}$ & $\epsilon_{s}$ & $\sigma_{s}$ & $\tau$ & $\epsilon_{r_{\text {avg }}}$ & $\sigma_{\text {avg }}$ \\
\hline \hline DI water & 4.55 & 77.11 & 0.0002 & $7.37 \mathrm{e}-12$ & 71.91 & 6.25 \\
\hline skin & 4.00 & 37.00 & 1.10 & $7.23 \mathrm{e}-12$ & 34.72 & 3.89 \\
\hline tumor & 3.99 & 54.00 & 0.70 & $7.0 \mathrm{e}-12$ & 50.74 & 4.82 \\
\hline breast \#1 & 7.00 & 10.00 & 0.15 & $7.0 \mathrm{e}-12$ & 9.80 & 0.40 \\
\hline breast \#2 & 6.57 & 16.29 & 0.23 & $7.0 \mathrm{e}-12$ & 15.66 & 1.03 \\
\hline breast \#3 & 6.14 & 22.57 & 0.31 & $7.0 \mathrm{e}-12$ & 21.50 & 1.66 \\
\hline breast \#4 & 5.71 & 28.86 & 0.39 & $7.0 \mathrm{e}-12$ & 27.35 & 2.30 \\
\hline breast \#5 & 5.28 & 35.14 & 0.46 & $7.0 \mathrm{e}-12$ & 33.20 & 2.92 \\
\hline
\end{tabular}

TABLE II

THERMAl CONSTANTS USED IN THE Numerical MODEL OF THE Bio-HEAT EQUation [31]-[37]

\begin{tabular}{c||c|c|c|c|c}
\hline \multirow{2}{*}{ Media } & \multicolumn{5}{c}{ Thermal Media Characteristics } \\
\cline { 2 - 6 } & $K\left[\frac{\mathrm{W}}{\mathrm{m}^{\circ}{ }^{\circ} \mathrm{C}}\right]$ & $C_{p}\left[\frac{\mathrm{J}}{\mathrm{kg} \cdot{ }^{\circ} \mathrm{C}}\right]$ & $\rho\left[\frac{\mathrm{kg}}{\mathrm{m}^{3}}\right]$ & $A_{0}\left[\frac{\mathrm{W}}{\mathrm{m}^{3}}\right]$ & $B\left[\frac{\mathrm{W}}{{ }^{\circ \mathrm{Cm}^{3}}}\right]$ \\
\hline \hline DI water & 0.6 & 4186 & 1000 & 0 & 0 \\
\hline skin & 0.376 & 3500 & 1100 & 1620 & 9100 \\
\hline tumor & 0.5641 & 3510 & 1020 & 480 & 2700 \\
\hline breast & 0.499 & 3550 & 1020 & 480 & 2700 \\
\hline
\end{tabular}

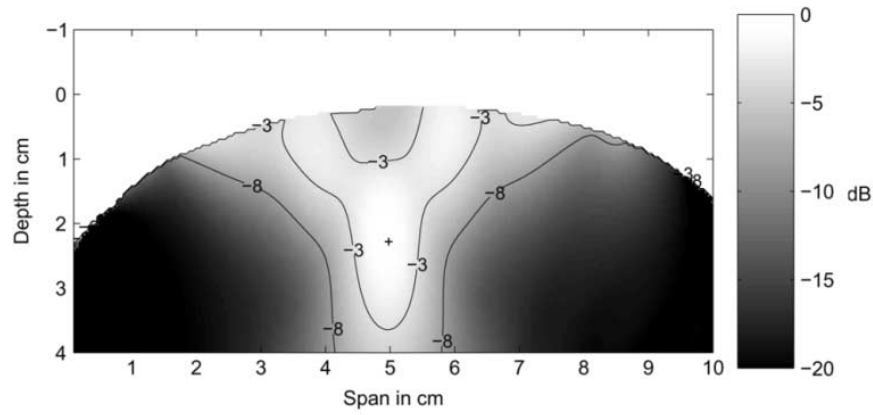

(a)

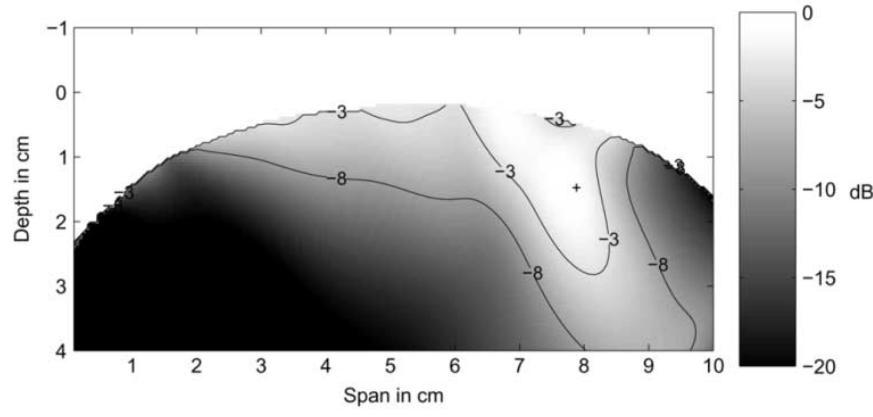

(b)

Fig. 4. Dissipated power density $(Q)$ in decibels calculated using the homogeneous FDTD breast model for the supine configuration. The beamformer is steered to the following locations: (a) $(5.0 \mathrm{~cm}, 3.0 \mathrm{~cm})$ and (b) $(8.0 \mathrm{~cm}, 2.0 \mathrm{~cm})$. The first and second coordinates in each pair represent span and depth, respectively. In each image, the maximum energy is denoted by a "+" marker.

prone (or face-down) position with the breast extending through an opening in the treatment table. In this position the antenna array encircles or in some other manner surrounds the pendulous breast. This configuration allows easier access to the full volume of the breast.

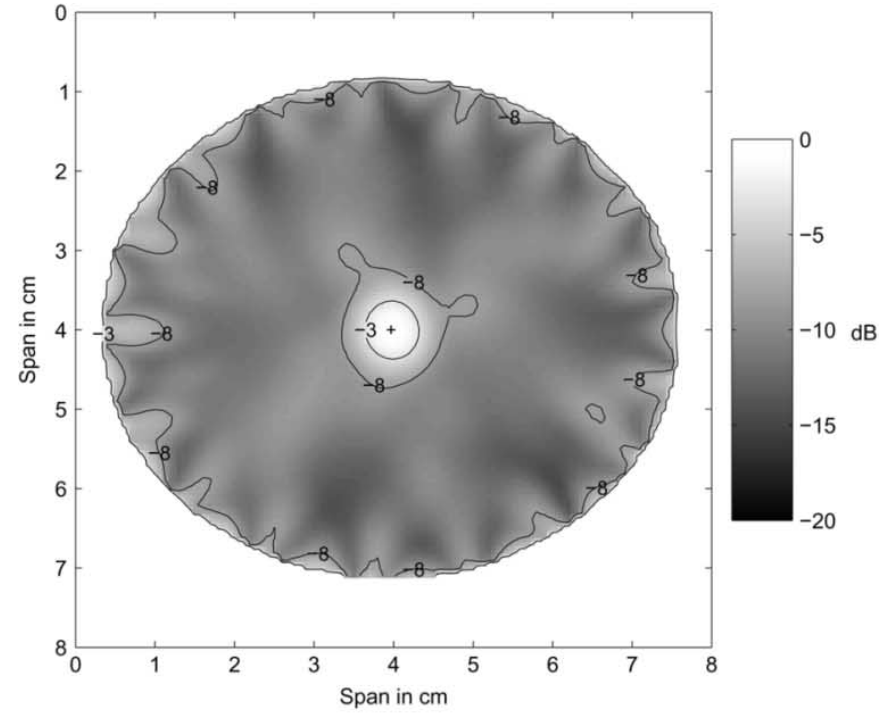

(a)

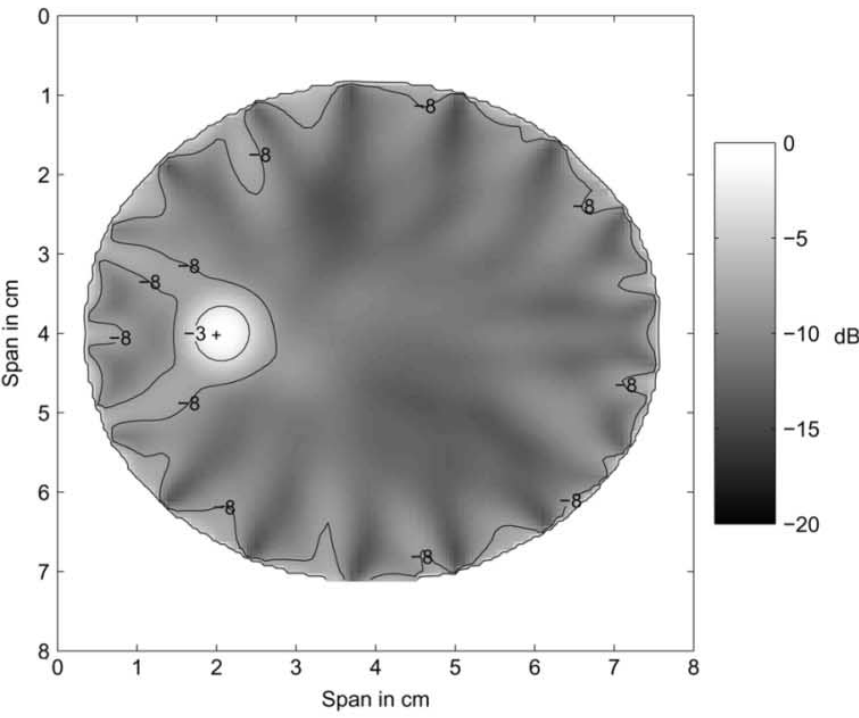

(b)

Fig. 5. Dissipated power density $(Q)$ in decibels calculated using the homogeneous FDTD breast model for the prone configuration. The beamformer is steered to the following locations: (a) $(4.0 \mathrm{~cm}, 4.0 \mathrm{~cm})$ and (b) $(2.0 \mathrm{~cm}, 4.0 \mathrm{~cm})$. The first and second coordinates in each pair both represent span. In each image, the maximum energy is denoted by a "+" marker.

To approximate the supine configuration in 2-D, we consider a sagittal plane through the breast with antennas positioned along the top of the breast, as shown in Fig. 3(a). The grayscale display of the interior of the breast shows the anatomically realistic variation of the permittivity within the breast, which is derived from the density variation of a high-resolution $(0.625 \mathrm{~mm} \times 0.625 \mathrm{~mm}$ pixel size $)$ breast MRI data set. Here, lighter regions represent higher dielectric-property values of denser fibroglandular tissue, while the darker regions represent lower dielectric-property values of less dense adipose tissue. To approximate the prone position in 2-D, we consider a coronal plane through the breast with antennas surrounding the breast, as shown in Fig. 3(b). We note that the between-plane resolution of the original 3-D MRI data set (1.5-mm spacing between MRI slices) is lower than the in-plane resolution. Since the in-plane scans correspond to the sagittal plane, it is the coronal 


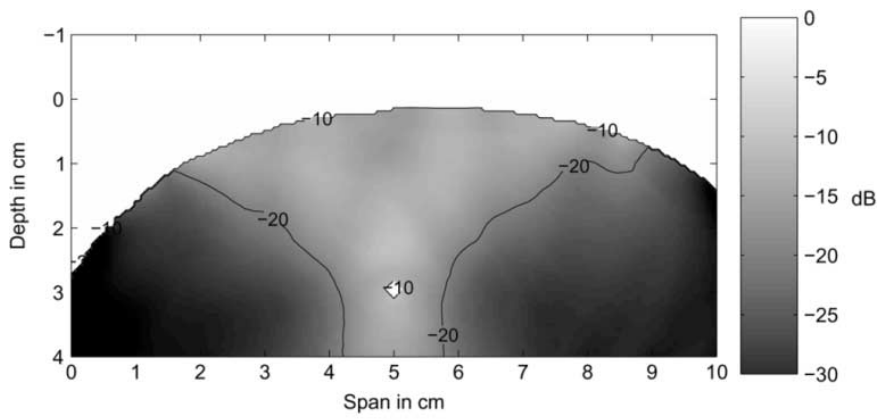

(a)

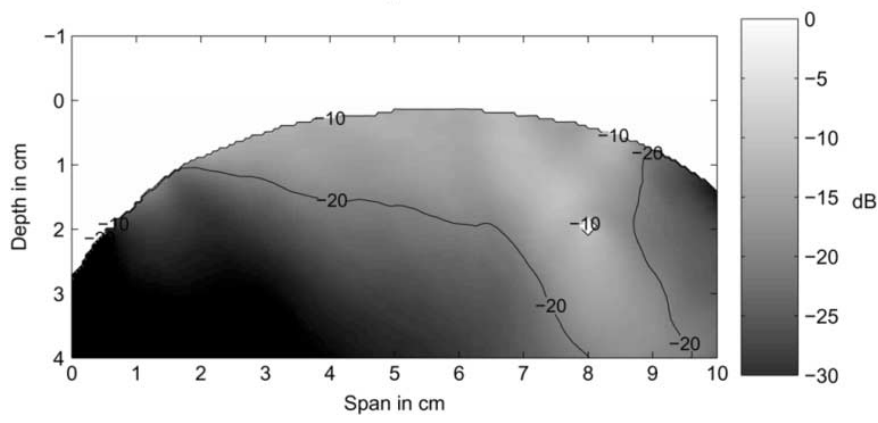

(b)

Fig. 6. $Q$ in decibels calculated using the heterogeneous FDTD breast model (breast \#1 with $\pm 10 \%$ variability) for the supine configuration. (a) Centered and (b) off-centered tumor location.

plane that is affected by the lower between-plane resolution. This explains why there is a slight decrease of clarity in the coronal image. The data from each MRI data set is interpolated to achieve the desired grid resolution for the EM model.

A 2-mm-diameter malignant tumor has been inserted into each breast model. In the sagittal model of Fig. 3(a), the tumor is shown in a central location in the breast at a depth of $3 \mathrm{~cm}$ below the surface of the breast. In the coronal model of Fig. 3(b), the tumor is also shown in a central location in the breast at a distance of 3-3.5 cm from the surface of the breast. Thus, each model is composed of the following four different media:

1) heterogeneous normal breast tissue;

2) skin;

3) malignant tumor;

4) deionized water.

\section{B. EM Model}

A 2-D TM ${ }_{z}$ FDTD-based EM model is used to investigate the effectiveness of our UWB space-time beamforming strategy for focusing energy at the site of the tumor. The FDTD EM model solves Maxwell's equations on a discrete spatial grid comprised of a numerical breast phantom and antenna array configuration shown in Fig. 3. The grid resolution used for these simulations is $0.5 \times 0.5 \mathrm{~mm}$. The dispersive tissue properties are incorporated in a manner similar to that presented in [28]. The dispersion characteristics are modeled using single-pole Debye dispersion equations of the following form:

$$
\epsilon_{r}=\epsilon_{\infty}+\frac{\epsilon_{s}-\epsilon_{\infty}}{1+j \omega \tau_{\circ}}-j \frac{\sigma_{s}}{\omega \epsilon_{\circ}} .
$$

Here, $\epsilon_{\infty}$ is the relative permittivity at infinite frequency, $\epsilon_{s}$ is the static relative permittivity, $\sigma_{s}$ is the static conductivity, and

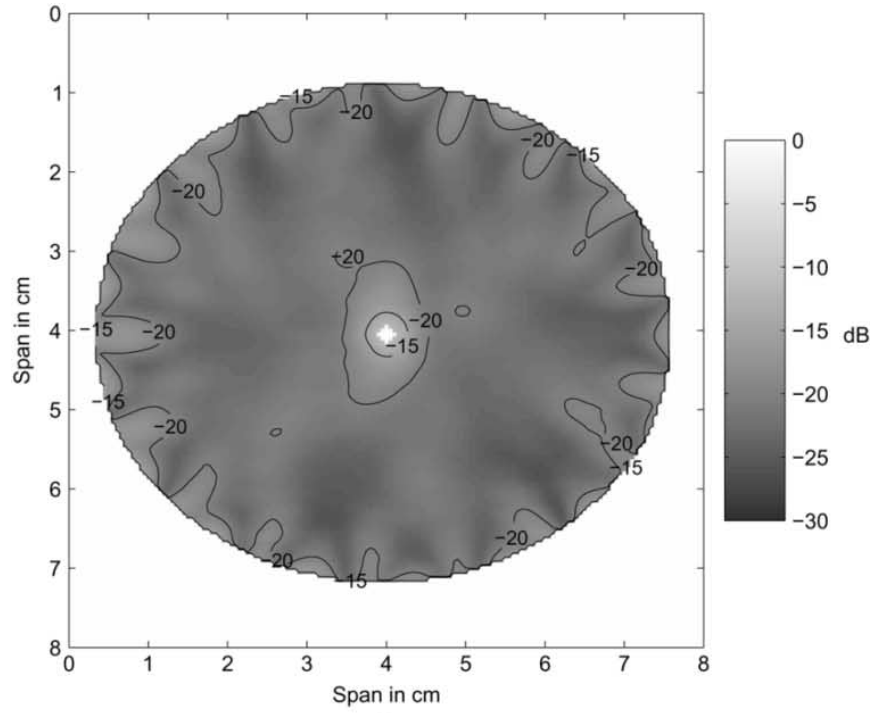

(a)

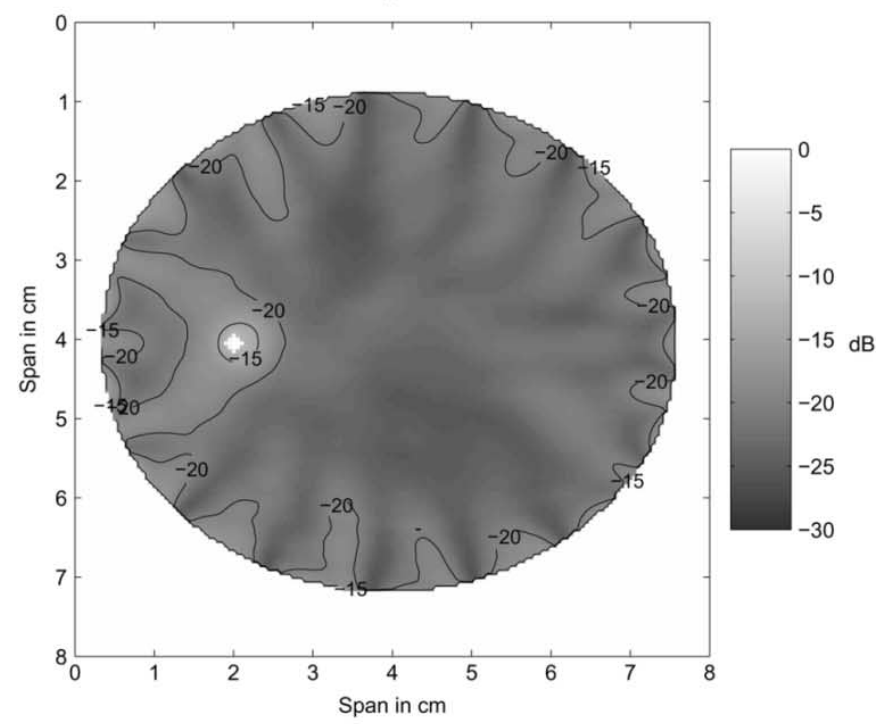

(b)

Fig. 7. $Q$ in decibels calculated using the heterogeneous FDTD breast model (breast \#1 with $\pm 10 \%$ variability) for the prone configuration. (a) Centered and (b) off-centered tumor location.

$\tau_{\circ}$ is the relaxation time constant. The specific Debye parameters for each of the materials are listed in Table I. Note that $\epsilon_{r_{\text {avg }}}$ and $\sigma_{\text {avg }}$ denote the values of permittivity and conductivity at $6 \mathrm{GHz}$ generated by the Debye model.

In practice, the average density of normal breast tissue and the degree of heterogeneity will vary from patient to patient with a certain margin. Therefore, in order to assess the robustness of our approach with respect to hypothetical variations in the characteristics of normal breast tissue, we consider five scenarios of normal breast tissue properties spanning the continuum from a breast model consisting of mostly fatty tissue (lower permittivity and conductivity) to that consisting of mostly fibroglandular tissue (higher permittivity and conductivity). Table I shows the five Debye parameter sets for the nominal dielectric properties of these normal breast tissue scenarios. Within each class of breast tissue density, we also consider five scenarios of variability about the average dielectric properties, ranging from $\pm 10 \%$ to $\pm 50 \%$. Thus, we 


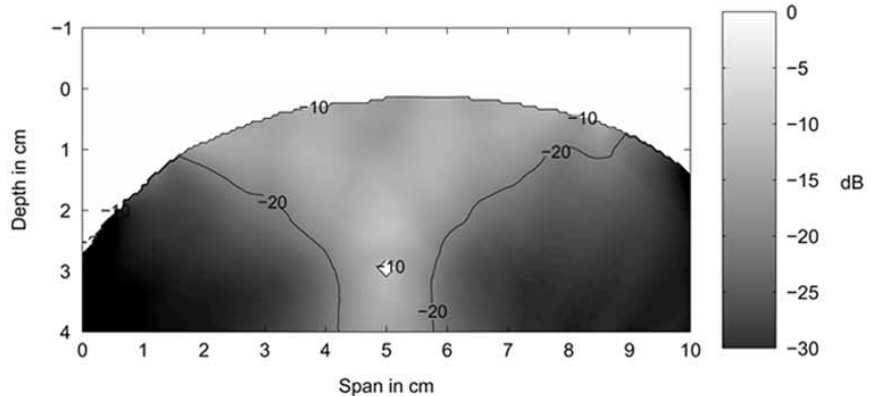

(a)

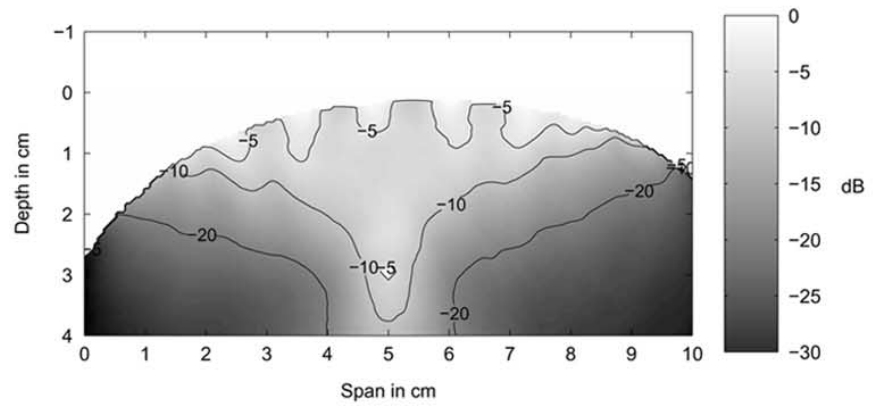

(c)

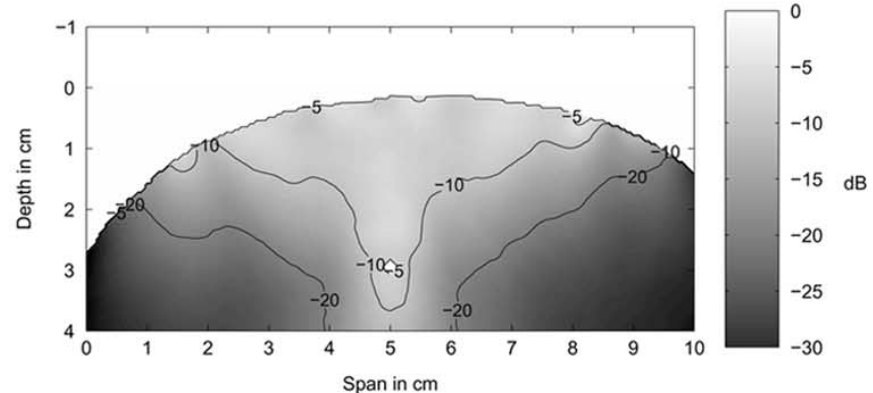

(b)

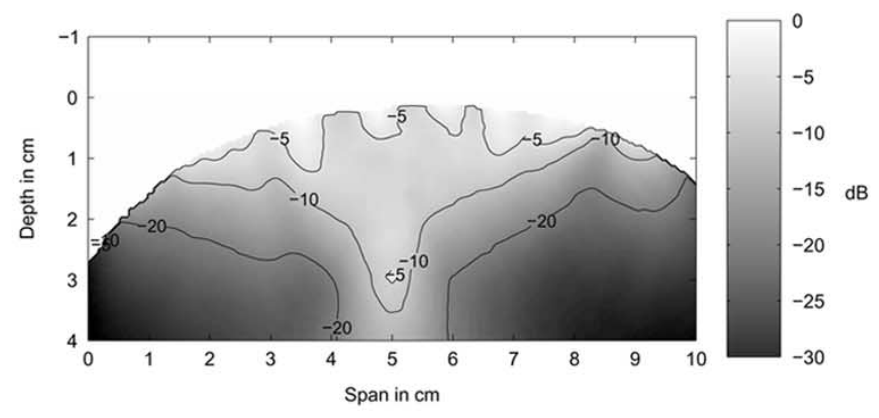

(d)

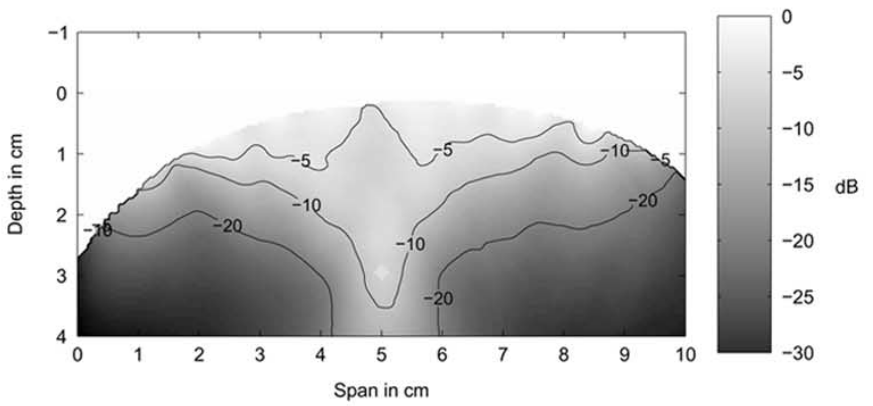

(e)

Fig. 8. $Q$ in decibels calculated using the heterogeneous FDTD breast model for the supine configuration as a function of normal breast tissue density. Here, the degree of heterogeneity (variability) is fixed at $\pm 10 \%$. The nominal dielectric properties of normal tissue are those of: (a) breast \#1 in Table I, (b) breast \#2, (c) breast \#3, (d) breast \#4, and (e) breast \#5.

obtain 25 different EM models with breast phantoms that range from mostly fatty tissue with little variability to very dense tissue with extreme heterogeneity. We note that these 25 cases were also used to test the robustness of the UWB space-time beamforming technique proposed for breast cancer detection [17].

The antennas are modeled as electric-current sources. We calculate the input power provided by each current source $J_{z \text {,source }}$ as follows:

$$
P_{\text {in }}=R \sum_{n=0}^{n_{\max }} E_{z}^{n} J_{z, \text { source }}^{n} \Delta x^{2} \Delta t\left[\frac{W}{m}\right]
$$

where $R$ is the assumed repetition rate of the pulses, $E_{z}^{n}$ and $J_{z \text {,source }}^{n}$ are the electric field and current density computed at each antenna location at time step $n, \Delta x$ is the grid cell size in the 2-D FDTD model, $\Delta t$ is the simulation time step, and $n_{\max }$ is the maximum number of time steps required to compute the response to a single UWB pulse radiated from each antenna. Since this is a 2-D simulation, the input power is normalized on a per-meter basis, yielding units of watts per meter.
EM power deposition is a well-accepted figure-of-merit for evaluating the effectiveness of the focusing strategy employed in hyperthermia. Therefore, using data computed during the FDTD simulation, we calculate the heating potential $(Q)$, i.e., the power dissipated per unit volume, as a function of location in the breast. We note that $Q$ is directly proportional to the specific absorption rate (SAR) with tissue density being the constant of proportionality. We calculate $Q$ using time-domain field quantities as follows [29]:

$$
Q_{i, j}=R \sum_{n=0}^{n_{\max }}\left(\sigma_{s_{i, j}}\left(E_{z_{i, j}}^{n}\right)^{2}+E_{z_{i, j}}^{n} \frac{\partial D_{z_{i, j}}^{n}}{\partial t}\right) \Delta t\left[\frac{W}{m^{3}}\right]
$$

where $i$ and $j$ are the computational lattice indices.

\section{Thermal Model}

Ultimately, the success of any hyperthermia approach depends on the resulting temperature distribution. We have constructed a relatively simple 2-D FDTD thermal model to obtain preliminary insights about the effectiveness of our proposed 


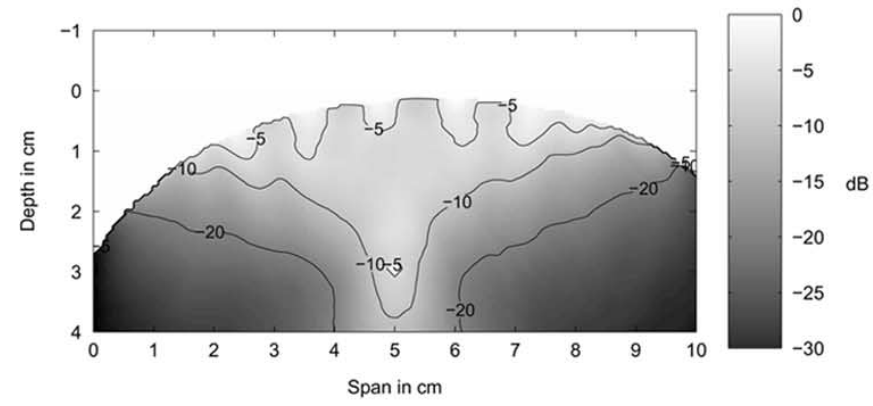

(a)

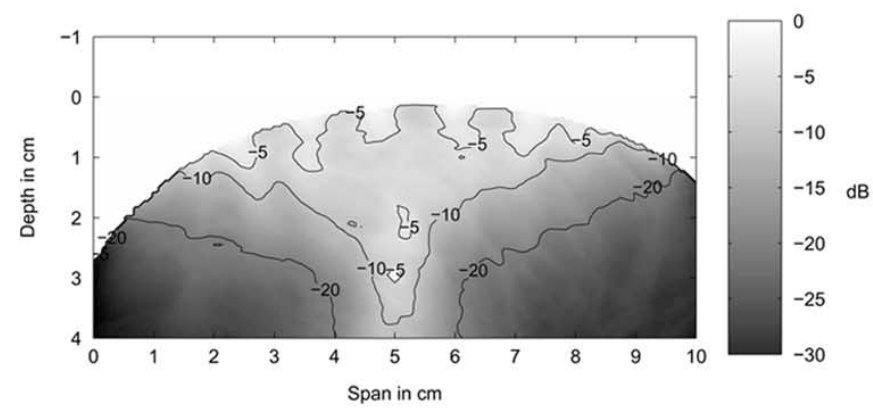

(c)

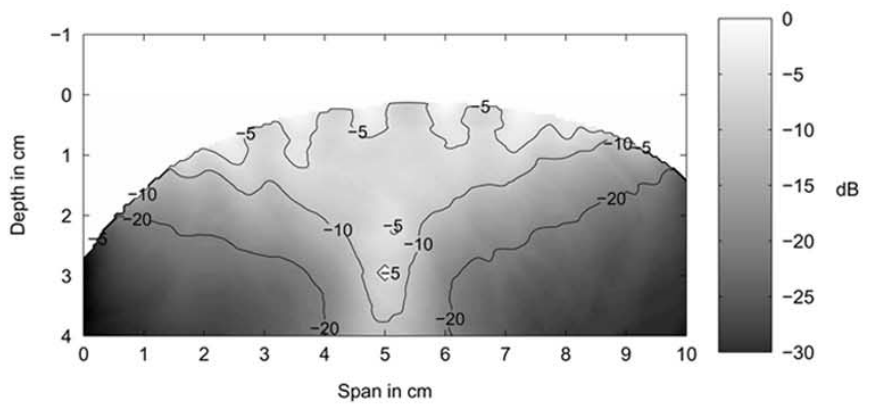

(b)

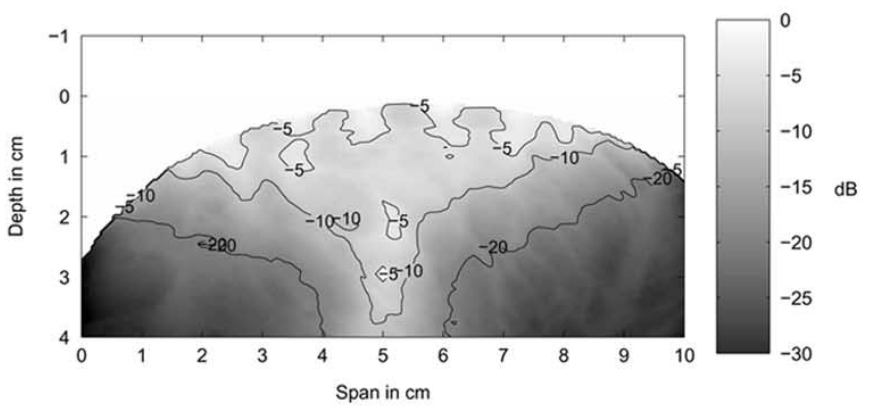

(d)

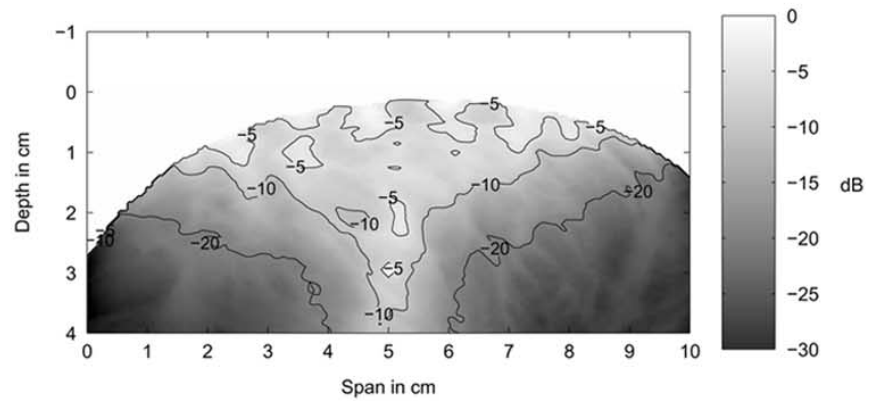

(e)

Fig. 9. $Q$ in decibels calculated using the heterogeneous FDTD breast model for the supine configuration as a function of normal breast tissue heterogeneity (variability). Here, the normal breast tissue density is fixed; the nominal dielectric properties are those of breast \#3. (a) $\pm 10 \%$. (b) $\pm 20 \%$. (c) $\pm 30 \%$. (d) $\pm 40 \%$. (e) $\pm 50 \%$.

UWB space-time hyperthermia technique in selectively raising the temperature of a specified region in the breast. The model is based on the well-known bio-heat equation

$$
\begin{aligned}
C_{p}(\mathbf{r}) \rho(\mathbf{r}) \frac{\partial T(\mathbf{r})}{\partial t}=\nabla \cdot( & K(\mathbf{r}) \nabla T(\mathbf{r}))+A_{0}(\mathbf{r})+Q(\mathbf{r}) \\
& -B(\mathbf{r})\left(T(\mathbf{r})-T_{B}\right)\left[\frac{W}{m^{3}}\right]
\end{aligned}
$$

which is discretized using the method of [31]. Here, $C_{p}$ is the specific heat, $\rho$ is the density, $K$ is the thermal conductivity, $A_{0}$ represents metabolic heat production, $Q$ is the deposited EM power density, $B$ is a constant representing the heat exchange mechanism due to capillary blood perfusion, and $T_{B}$ is the blood temperature (assumed to be at body temperature). A discussion of the thermal parameters and their role in the bio-heat equation is found in [31]. The thermal model consists of the same four media as the EM model and utilizes the same computational lattice. Thermal property data for the different elements are shown in Table II. All values have been taken from the literature when possible. Where we have been unable to find values specific to the breast, thermal constants have been approximated using values for other tissue types. Tumor density, metabolic heat generation, and the blood perfusion constant have been set to known values for breast tissue. The breast perfusion constant and metabolic heat generation have been set to a value determined for muscle. This model utilizes convective boundary conditions at the skin surface to simulate chilled water cooling. A constant temperature boundary condition of $37^{\circ}$ within the chest wall is used in the sagittal simulation. All simulations are run to steady-state conditions; therefore, basal temperature distributions are automatically accounted for.

\section{Simulation Results}

Here, we begin with an investigation of our focusing approach using a simple homogeneous breast model surrounded by the coupling medium of deionized water to examine the accuracy and focusing ability in a scenario that closely corresponds to the design assumptions. In Section IV-B, we examine the EM focusing effectiveness in an anatomically realistic breast model. This model includes the scattering effects of the skin and the heterogeneity of the breast associated with normal glandular and 


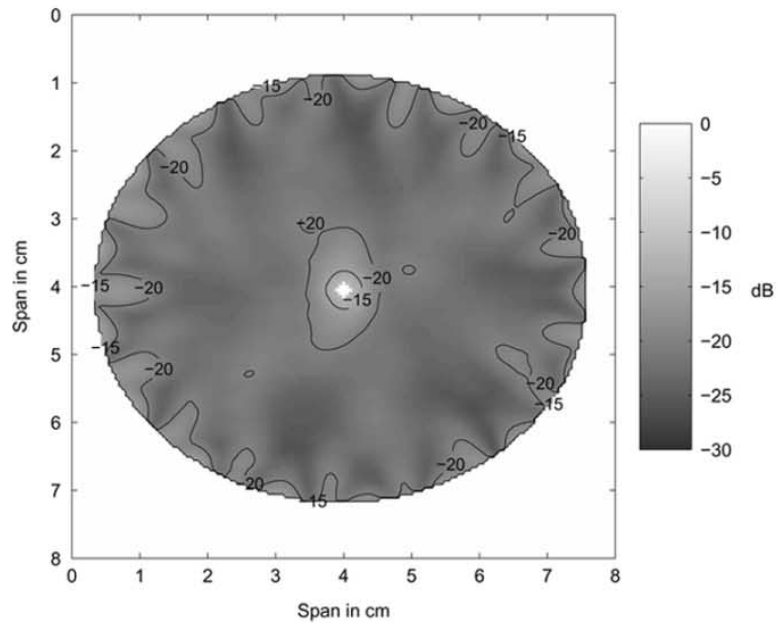

(a)

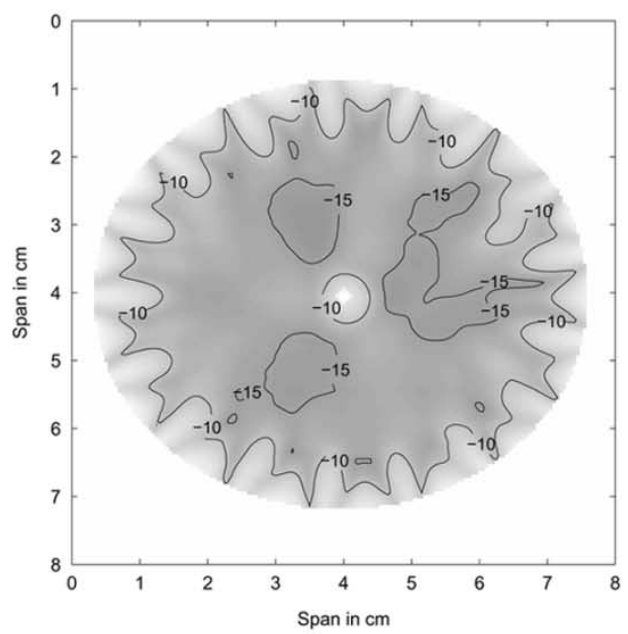

(c)

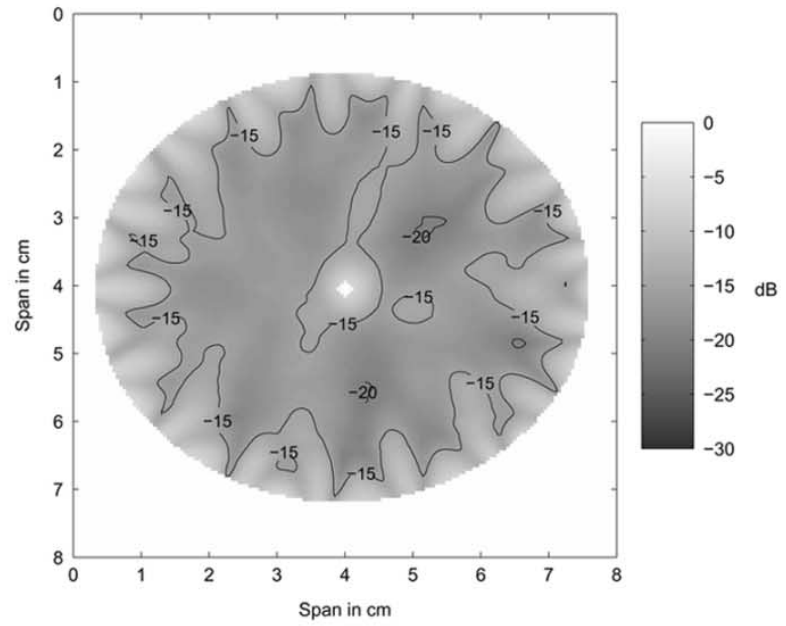

(b)

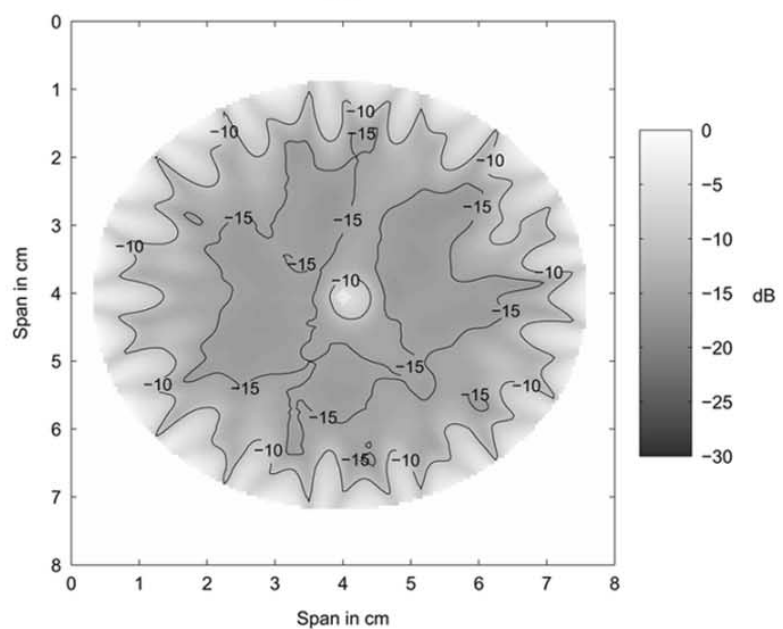

(d)

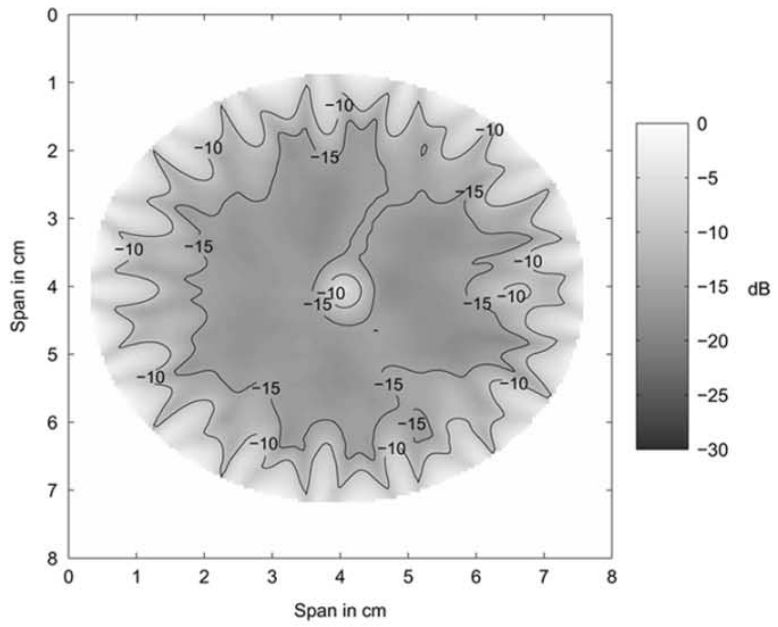

(e)

Fig. 10. $Q$ in decibels calculated using the heterogeneous FDTD breast model for the prone configuration as a function of normal breast tissue density. Here, the degree of heterogeneity (variability) is fixed at $\pm 10 \%$. The nominal dielectric properties of normal tissue are those of: (a) breast \#1 in Table I, (b) breast \#2, (c) breast \#3, (d) breast \#4, and (e) breast \#5.

adipose tissue. The presence of the tumor also introduces a scattering effect and a local increase in the power density due to the higher conductivity of the tumor. Section IV-C uses a simple thermal model to illustrate the temperature distribution resulting from one of the power density distributions.

\section{A. Beamformer Power Deposition in a Homogeneous Breast Model}

The design strategy presented in Section II assumes a homogeneous propagation model so here we evaluate focusing on the presence of minimal errors between the assumed and actual 


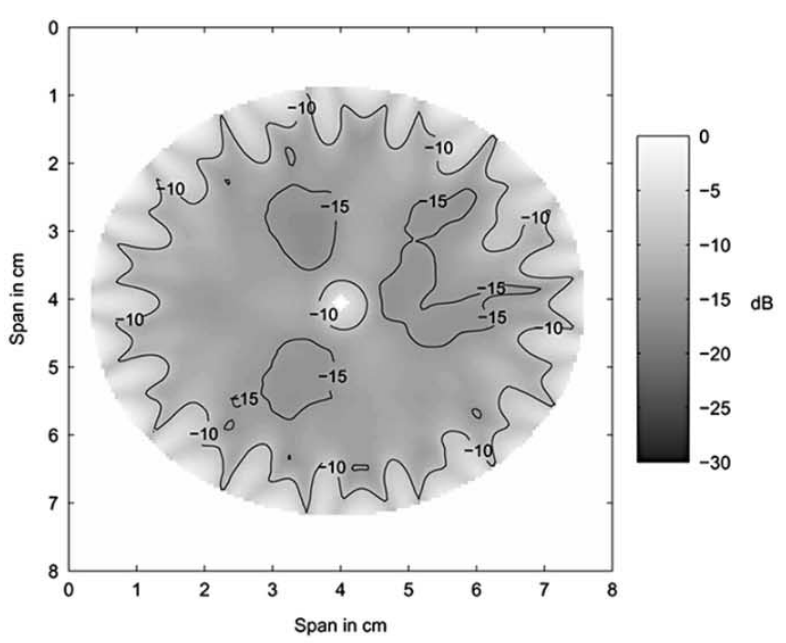

(a)

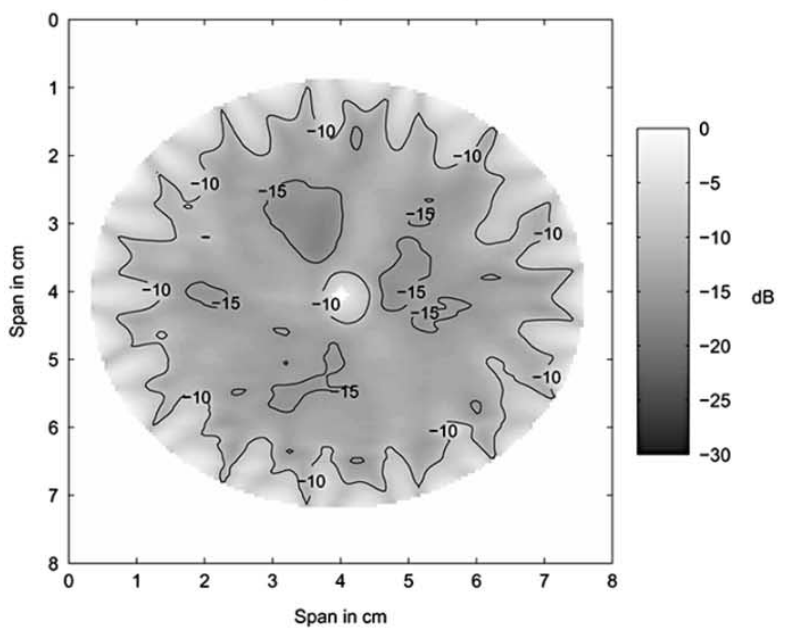

(c)

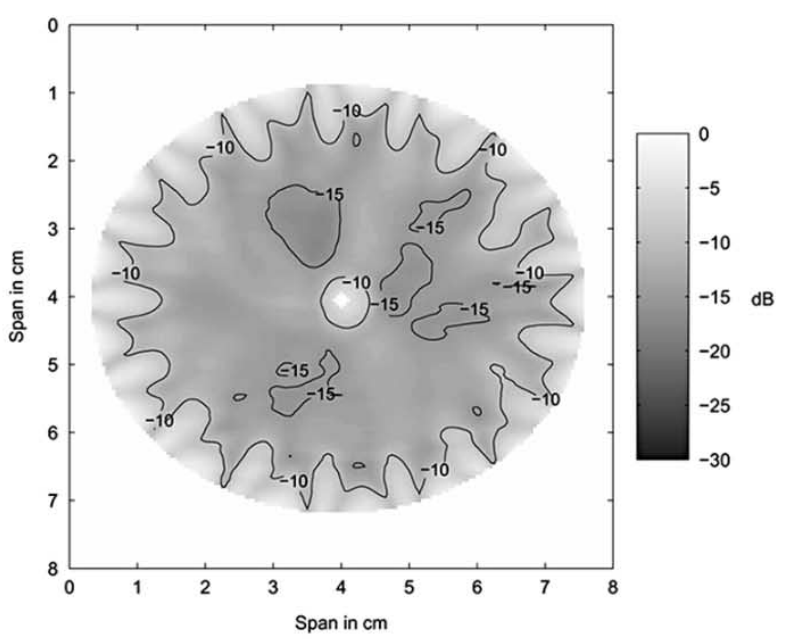

(b)

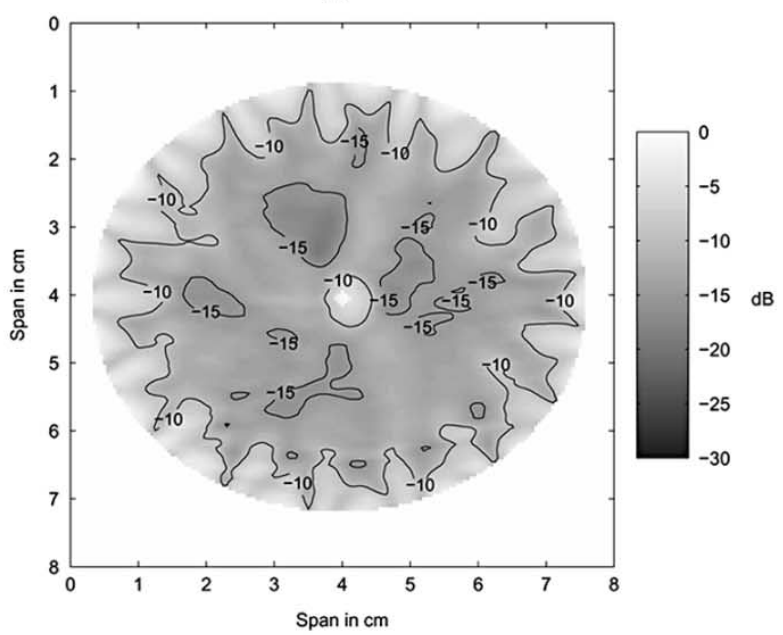

(d)

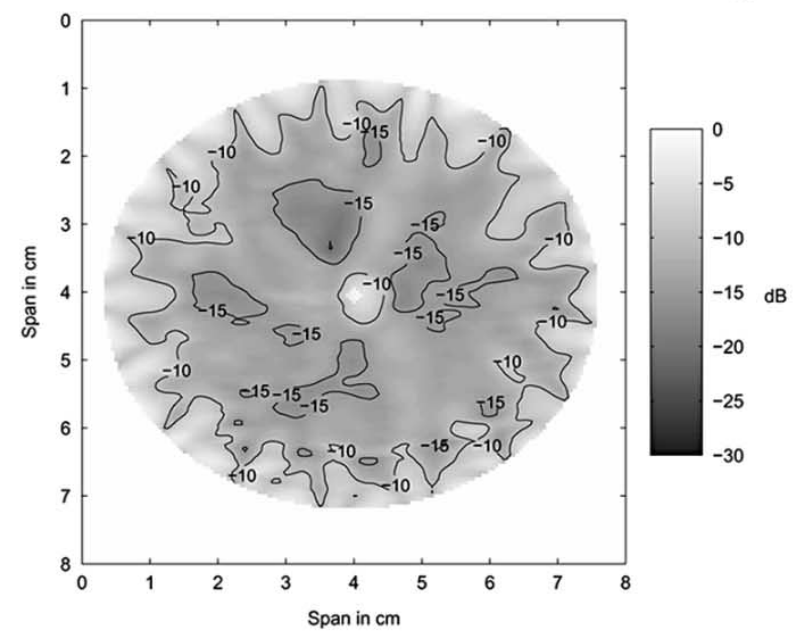

(e)

Fig. 11. $Q$ in decibels calculated using the heterogeneous FDTD breast model for the prone configuration as a function of normal breast tissue heterogeneity (variability). Here, the normal breast tissue density is fixed; the nominal dielectric properties are those of breast \#3. (a) $\pm 10 \%$. (b) $\pm 20 \%$. (c) $\pm 30 \%$. (d) $\pm 40 \%$. (e) $\pm 50 \%$.

propagation paths. This investigation also permits the evaluation of focusing under conditions where there is no dielectric-properties contrast between the heating zone and surrounding tissue. For this investigation, the heterogeneity, skin layer, and tumor shown in Fig. 3 are removed from the FDTD model. The dis- persive properties of deionized water and breast \#1, shown in Table I, are used in the FDTD simulation.

Fig. 4 illustrates the focusing capability of the beamformer for the supine configuration. In Fig. 4(a), the beamformer is designed to focus at $\mathbf{r}_{0}=(5.0,3.0)$. The maximum $Q$ occurs at 
TABLE III

Ratio of Average Breast $Q$ to Average Tumor $Q$ in the Region Within a 1 -cm Radius of the Tumor for the Supine Configuration

\begin{tabular}{c||c|c|c|c|c}
\hline \multicolumn{1}{c||}{} & \multicolumn{5}{c}{ Breast/Tumor Q for the supine configuration } \\
\cline { 2 - 6 } Variability & $\begin{array}{c}\varepsilon_{r_{\text {avg }}}=9.8 \\
\sigma_{\text {avg }}=0.4 \mathrm{~S} / \mathrm{m}\end{array}$ & $\begin{array}{c}\varepsilon_{r_{\text {avg }}}=15.7 \\
\sigma_{\text {avg }}=1.0 \mathrm{~S} / \mathrm{m}\end{array}$ & $\begin{array}{c}\varepsilon_{r_{\text {avg }}}=21.5 \\
\sigma_{\text {avg }}=1.7 \mathrm{~S} / \mathrm{m}\end{array}$ & $\begin{array}{c}\varepsilon_{r_{\text {avg }}}=27.3 \\
\sigma_{\text {avg }}=2.3 \mathrm{~S} / \mathrm{m}\end{array}$ & $\begin{array}{c}\varepsilon_{r_{\text {avg }}}=33.2 \\
\sigma_{\text {avg }}=2.9 \mathrm{~S} / \mathrm{m}\end{array}$ \\
\hline \hline $\pm 10 \%$ & $-13.7 \mathrm{~dB}$ & $-9.8 \mathrm{~dB}$ & $-7.7 \mathrm{~dB}$ & $-6.3 \mathrm{~dB}$ & $-5.2 \mathrm{~dB}$ \\
\hline $\pm 20 \%$ & $-13.7 \mathrm{~dB}$ & $-9.8 \mathrm{~dB}$ & $-7.8 \mathrm{~dB}$ & $-6.3 \mathrm{~dB}$ & $-5.3 \mathrm{~dB}$ \\
\hline $\pm 30 \%$ & $-13.8 \mathrm{~dB}$ & $-9.9 \mathrm{~dB}$ & $-7.8 \mathrm{~dB}$ & $-6.4 \mathrm{~dB}$ & $-5.1 \mathrm{~dB}$ \\
\hline $\pm 40 \%$ & $-13.8 \mathrm{~dB}$ & $-9.9 \mathrm{~dB}$ & $-7.8 \mathrm{~dB}$ & $-6.4 \mathrm{~dB}$ & $-5.3 \mathrm{~dB}$ \\
\hline $\pm 50 \%$ & $-13.8 \mathrm{~dB}$ & $-9.9 \mathrm{~dB}$ & $-7.7 \mathrm{~dB}$ & $-6.3 \mathrm{~dB}$ & $-5.2 \mathrm{~dB}$ \\
\hline
\end{tabular}

TABLE IV

Ratio of Average Surface $Q$ to Average Tumor $Q$ In the Region Within $0.5 \mathrm{~cm}$ of the Skin For the Supine Configuration

\begin{tabular}{c||c|c|c|c|c}
\hline \multirow{2}{*}{ Variability } & \multicolumn{5}{c}{ Surface/Tumor Q for the supine configuration } \\
\cline { 2 - 6 } & $\begin{array}{c}\varepsilon_{r_{\text {avg }}}=9.8 \\
\sigma_{\text {avg }}=0.4 \mathrm{~S} / \mathrm{m}\end{array}$ & $\begin{array}{c}\varepsilon_{r_{\text {avg }}}=15.7 \\
\sigma_{\text {avg }}=1.0 \mathrm{~S} / \mathrm{m}\end{array}$ & $\begin{array}{c}\varepsilon_{r_{\text {avg }}}=21.5 \\
\sigma_{\text {avg }}=1.7 \mathrm{~S} / \mathrm{m}\end{array}$ & $\begin{array}{c}\varepsilon_{r_{\text {avg }}}=27.3 \\
\sigma_{\text {avg }}=2.3 \mathrm{~S} / \mathrm{m}\end{array}$ & $\begin{array}{c}\varepsilon_{r_{\text {avg }}}=33.2 \\
\sigma_{\text {avg }}=2.9 \mathrm{~S} / \mathrm{m}\end{array}$ \\
\hline \hline $\pm 10 \%$ & $-13.7 \mathrm{~dB}$ & $-6.9 \mathrm{~dB}$ & $-2.9 \mathrm{~dB}$ & $-0.2 \mathrm{~dB}$ & $1.6 \mathrm{~dB}$ \\
\hline $\pm 20 \%$ & $-13.8 \mathrm{~dB}$ & $-7.0 \mathrm{~dB}$ & $-3.0 \mathrm{~dB}$ & $-0.3 \mathrm{~dB}$ & $1.5 \mathrm{~dB}$ \\
\hline $\pm 30 \%$ & $-13.9 \mathrm{~dB}$ & $-7.0 \mathrm{~dB}$ & $-3.0 \mathrm{~dB}$ & $-0.4 \mathrm{~dB}$ & $1.7 \mathrm{~dB}$ \\
\hline $\pm 40 \%$ & $-13.9 \mathrm{~dB}$ & $-7.0 \mathrm{~dB}$ & $-3.0 \mathrm{~dB}$ & $-0.4 \mathrm{~dB}$ & $1.6 \mathrm{~dB}$ \\
\hline $\pm 50 \%$ & $-13.9 \mathrm{~dB}$ & $-6.9 \mathrm{~dB}$ & $-2.8 \mathrm{~dB}$ & $-0.3 \mathrm{~dB}$ & $1.8 \mathrm{~dB}$ \\
\hline
\end{tabular}

TABLE V

Ratio of Average Breast $Q$ to Average Tumor $Q$ in the Region Within a 1 -cm Radius of the Tumor for the Prone Configuration

\begin{tabular}{c||c|c|c|c|c}
\hline \multicolumn{1}{c||}{} & \multicolumn{5}{c}{ Breast/Tumor Q for the prone configuration } \\
\cline { 2 - 6 } Variability & $\begin{array}{c}\varepsilon_{r_{\text {avg }}}=9.8 \\
\sigma_{\text {avg }}=0.4 \mathrm{~S} / \mathrm{m}\end{array}$ & $\begin{array}{c}\varepsilon_{r_{\text {avg }}}=15.7 \\
\sigma_{\text {avg }}=1.0 \mathrm{~S} / \mathrm{m}\end{array}$ & $\begin{array}{c}\varepsilon_{r_{\text {avg }}}=21.5 \\
\sigma_{\text {avg }}=1.7 \mathrm{~S} / \mathrm{m}\end{array}$ & $\begin{array}{c}\varepsilon_{r_{\text {avg }}}=27.3 \\
\sigma_{\text {avg }}=2.3 \mathrm{~S} / \mathrm{m}\end{array}$ & $\begin{array}{c}\varepsilon_{r_{\text {avg }}}=33.2 \\
\sigma_{\text {avg }}=2.9 \mathrm{~S} / \mathrm{m}\end{array}$ \\
\hline \hline $\pm 10 \%$ & $-17.9 \mathrm{~dB}$ & $-13.7 \mathrm{~dB}$ & $-11.7 \mathrm{~dB}$ & $-9.9 \mathrm{~dB}$ & $-9.2 \mathrm{~dB}$ \\
\hline $\pm 20 \%$ & $-17.8 \mathrm{~dB}$ & $-13.4 \mathrm{~dB}$ & $-11.4 \mathrm{~dB}$ & $-9.3 \mathrm{~dB}$ & $-8.7 \mathrm{~dB}$ \\
\hline $\pm 30 \%$ & $-17.5 \mathrm{~dB}$ & $-12.9 \mathrm{~dB}$ & $-11.0 \mathrm{~dB}$ & $-8.8 \mathrm{~dB}$ & $-8.1 \mathrm{~dB}$ \\
\hline $\pm 40 \%$ & $-17.1 \mathrm{~dB}$ & $-12.5 \mathrm{~dB}$ & $-10.6 \mathrm{~dB}$ & $-8.1 \mathrm{~dB}$ & $-7.5 \mathrm{~dB}$ \\
\hline $\pm 50 \%$ & $-16.8 \mathrm{~dB}$ & $-12.0 \mathrm{~dB}$ & $-9.9 \mathrm{~dB}$ & $-7.6 \mathrm{~dB}$ & $-6.8 \mathrm{~dB}$ \\
\hline
\end{tabular}

$(5.0,2.3)$, as indicated by the "+" marker. At $\mathbf{r}_{0}$, the $Q$ is $1.5 \mathrm{~dB}$ less than the maximum. The image shows that at a lateral distance of $1.0 \mathrm{~cm}$ away from the design location, the $Q$ is $8 \mathrm{~dB}$ below the maximum. Fig. 4(b) depicts $Q$ when the focus location is off-center at $\mathbf{r}_{0}=(8.0,2.0)$. Here, the maximum $Q$ is at $(7.9,1.5)$. Once again, the location of the maximum occurs at a shallower location than the desired spot. This is expected since the beamformer focus is biased toward shallower regions due to the need to compensate for attenuation. Similar effects are observed in receive beamforming [17], where attenuation can cause scatterers to appear deeper than their true locations. Fig. 4(a) and (b) indicates that the beamformer is more selective in the lateral dimension than the depth dimension. This is inherent to the supine configuration since the array aperture primarily spans the lateral dimension and has a very small projection in the depth dimension due to the flattened nature of the breast.

Power density deposition images for the prone configuration are shown in Fig. 5. Fig. 5(a) illustrates the case when the beamformer is designed to focus energy at $(4.0,4.0)$, while the focus location in Fig. 5(b) is $(2.0,4.0)$. The maximum $Q$ occurs at the designed focus location in both cases, and the $-3-\mathrm{dB}$ radius of $Q$ is approximately $0.5 \mathrm{~cm}$. In the prone configuration, the array aperture has approximately equal spans in both dimensions so the $Q$ distribution lacks the asymmetry observed in the supine configuration.

\section{B. Beamformer Power Deposition in a Heterogeneous Breast Model}

The MRI-derived breast model used here includes the effects of the skin, tumor, and realistic tissue heterogeneity. This allows us to assess whether the simple homogeneous propagation model used in beamformer design produces satisfactory results in realistic scenarios. It also permits the evaluation of focusing under conditions where there is a dielectric-properties contrast between the heating zone and surrounding tissue. Figs. 6 and 7 display $Q$ for the supine and prone configurations, respectively, with the same focus locations as Figs. 4 and 5. Note that more selective focusing is obtained using the anatomically realistic breast model (Figs. 6 and 7) than the homogeneous model (Figs. 4 and 5). This is because the tumor has higher conductivity than the surrounding breast tissue and $Q$ is proportional to conductivity [see (11)].

Fig. 8 shows $Q$ for the supine configuration as the nominal dielectric properties of normal breast tissue are varied from low (breast \#1) to high (breast \#5). All of these cases assume the maximum density variation in the MRI maps to a $\pm 10 \%$ variation about the nominal dielectric properties. Here, the tumor 
TABLE VI

Ratio of Average Surface $Q$ to Average Tumor $Q$ in the Region Within $0.5 \mathrm{~cm}$ of the Skin for the Prone Configuration

\begin{tabular}{c||c|c|c|c|c}
\hline \multicolumn{1}{c||}{} & \multicolumn{5}{c}{ Surface/Tumor Q for the prone configuration } \\
\cline { 2 - 6 } Variability & $\begin{array}{c}\varepsilon_{r_{\text {avg }}}=9.8 \\
\sigma_{\text {avg }}=0.4 \mathrm{~S} / \mathrm{m}\end{array}$ & $\begin{array}{c}\varepsilon_{r_{\text {avg }}}=15.7 \\
\sigma_{\text {avg }}=1.0 \mathrm{~S} / \mathrm{m}\end{array}$ & $\begin{array}{c}\varepsilon_{r_{\text {avg }}}=21.5 \\
\sigma_{\text {avg }}=1.7 \mathrm{~S} / \mathrm{m}\end{array}$ & $\begin{array}{c}\varepsilon_{r_{\text {avg }}}=27.3 \\
\sigma_{\text {avg }}=2.3 \mathrm{~S} / \mathrm{m}\end{array}$ & $\begin{array}{c}\varepsilon_{r_{\text {avg }}}=33.2 \\
\sigma_{\text {avg }}=2.9 \mathrm{~S} / \mathrm{m}\end{array}$ \\
\hline \hline $\pm 10 \%$ & $-19.3 \mathrm{~dB}$ & $-10.6 \mathrm{~dB}$ & $-6.1 \mathrm{~dB}$ & $-2.5 \mathrm{~dB}$ & $-0.7 \mathrm{~dB}$ \\
\hline $\pm 20 \%$ & $-19.3 \mathrm{~dB}$ & $-10.4 \mathrm{~dB}$ & $-5.9 \mathrm{~dB}$ & $-2.1 \mathrm{~dB}$ & $-0.3 \mathrm{~dB}$ \\
\hline $\pm 30 \%$ & $-19.2 \mathrm{~dB}$ & $-10.1 \mathrm{~dB}$ & $-5.6 \mathrm{~dB}$ & $-1.8 \mathrm{~dB}$ & $0.1 \mathrm{~dB}$ \\
\hline $\pm 40 \%$ & $-19.1 \mathrm{~dB}$ & $-9.7 \mathrm{~dB}$ & $-5.3 \mathrm{~dB}$ & $-1.2 \mathrm{~dB}$ & $0.7 \mathrm{~dB}$ \\
\hline $\pm 50 \%$ & $-18.8 \mathrm{~dB}$ & $-9.3 \mathrm{~dB}$ & $-4.7 \mathrm{~dB}$ & $-0.7 \mathrm{~dB}$ & $1.3 \mathrm{~dB}$ \\
\hline
\end{tabular}

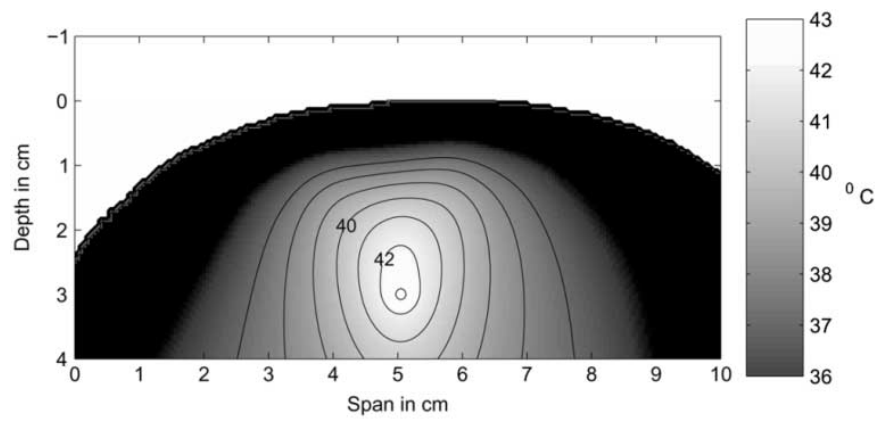

(a)

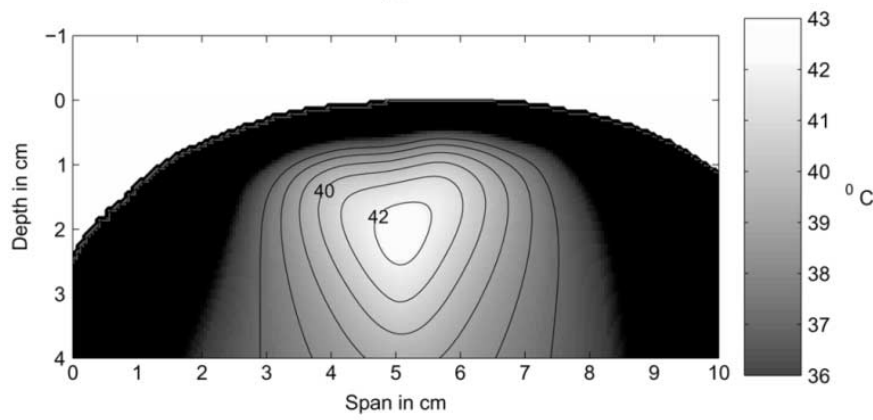

(b)

Fig. 12. Temperature distribution calculated using the FDTD thermal mode for the supine configuration with the power deposition pattern of: (a) Fig. 8(a) (breast \#1 with $\pm 10 \%$ variability) and (b) Fig. $8(e)$ (breast \#5 with $\pm 10 \%$ variability).

is centered at $(5.0,3.0)$. As shown in Fig. 8, better focusing is obtained in breast phantoms with lower normal-tissue permittivity and conductivity. As the density of the breast increases, attenuation in normal breast tissue increases and the contrast between malignant and normal breast tissue decreases. Consequently, more energy is deposited near the surface and the focus at the tumor spreads. Fig. 9 shows $Q$ as the variability changes from $\pm 10 \%$ to $\pm 50 \%$ about the nominal dielectric properties of breast \#3. As is evident in this figure, there is relatively little difference between the results as the dielectric-properties variability increases from low to high, indicating that our focusing approach is relatively unaffected by the degree of heterogeneity of the breast. Figs. 10 and 11 repeat this robustness study for the prone configuration. Here, the tumor is centered at $(4.0,4.0)$. As in the supine configuration, increasing the nominal dielectric properties (density) of normal tissue leads to increased power deposition near the surface and less selective focusing, while increasing the variability (heterogeneity) has only a minor effect on $Q$.

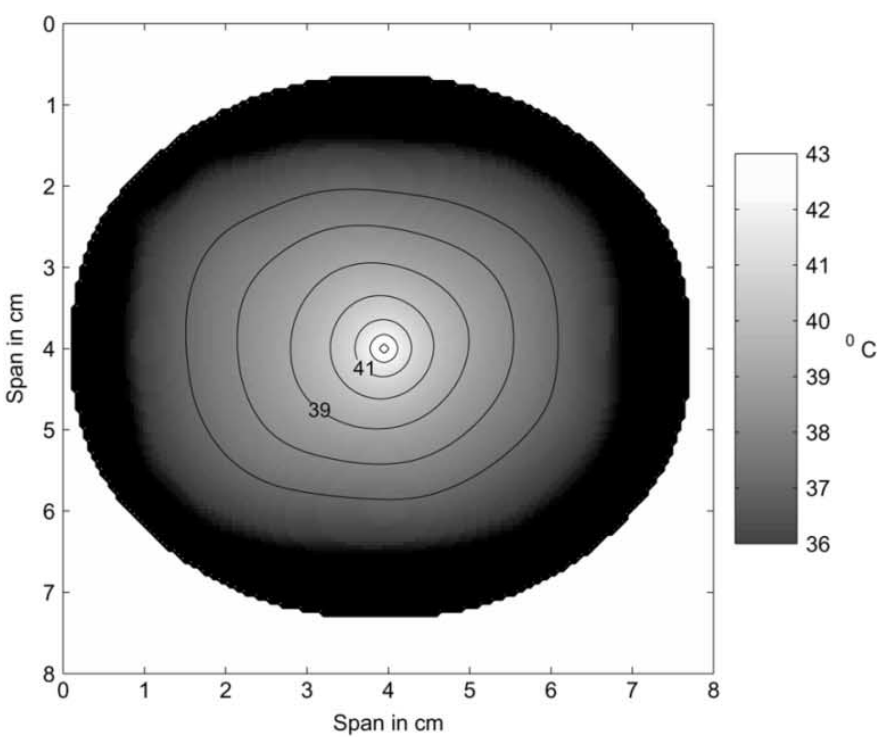

(a)

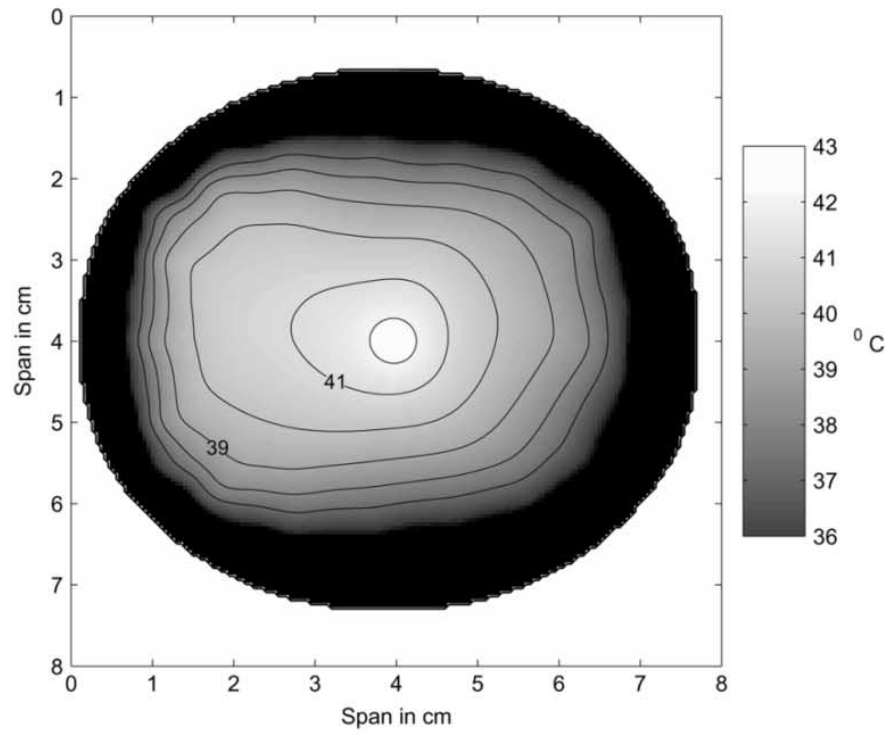

(b)

Fig. 13. Temperature distribution calculated using the FDTD thermal model for the prone configuration with the power deposition pattern of Fig. 10(a) (breast \#1 with $\pm 10 \%$ variability) and (b) Fig. 10(e) (breast \#5 with $\pm 10 \%$ variability).

We have chosen to quantitatively summarize the results in Figs. 8-11 using two metrics of focusing selectivity: $\left(Q_{\text {ave }}\right.$ (breast $\left.)\right) /\left(\mathrm{Q}_{\text {ave }}(\right.$ tumor $\left.)\right)$, where the average breast $Q$ is determined from data in the region within a $1-\mathrm{cm}$ radius of 
the center of the tumor, and $\left(Q_{\text {ave }}(\right.$ surface $\left.)\right) /\left(\mathrm{Q}_{\mathrm{ave}}(\right.$ tumor $\left.)\right)$, where the average surface $Q$ is determined from data in a 0.5 -cm-thick layer beneath the skin/breast interface. Smaller values for both metrics indicate more selective focusing at the site of the tumor and less power deposition in the surrounding normal tissue.

Tables III-VI show the two metrics for the cases presented in Figs. 8-11. As expected from the trends observed in these figures, the metrics are more sensitive to increases in nominal dielectric properties (density) of normal breast tissue and relatively insensitive to the level of heterogeneity within the breast. The selectivity metrics for the prone configuration are generally several decibels smaller than those for the supine configuration, which reflects the improvement in focusing selectivity for the prone configuration depicted in Figs. 8-11.

\section{2-D FDTD Thermal Simulation Results}

Figs. 12 and 13 show the temperature distribution within the breast for supine and prone configurations, respectively. In both cases, the thermal model is sourced with the power deposition data computed for either breast phantom \#1 with $\pm 10 \%$ variability or breast \#5 with $\pm 10 \%$ variability. The input power to the antennas and the temperature of the chilled cooling water was varied for each case, with total input power/centimeter varying from 16.8 to $57.3 \mathrm{~W} / \mathrm{cm}$ and water temperatures varying from $15{ }^{\circ} \mathrm{C}$ to $30{ }^{\circ} \mathrm{C}$. Hyperthermia generally requires elevation of the target region to temperatures from $42{ }^{\circ} \mathrm{C}$ to $43{ }^{\circ} \mathrm{C}$, while maintaining the temperatures of healthy tissue regions below $42{ }^{\circ} \mathrm{C}$. These figures suggest that our approach can provide the temperature selectivity needed for effective hyperthermia treatment in a variety of breast tissue environments.

\section{CONCLUSION}

An investigation into the feasibility of using a UWB space-time beamforming microwave system for breast hyperthermia has been reported. A multiantenna system with FIR filters in each channel is used to compensate for the dispersive properties of breast tissue and provide constructive interference of the transmitted UWB pulses at a specified location in the breast. Initial feasibility studies of the effectiveness of this focusing method are performed using 2-D FDTD EM and thermal simulations. The results of these simulations show excellent EM focusing ability and suggest that the necessary temperature gradients required for effective hyperthermia may be achieved with this method. More advanced models are being developed to further investigate and validate the preferential heating capability of the technique.

\section{REFERENCES}

[1] C. C. Vernon, J. W. Hand, S. B. Field, D. Machin, J. B. Whaley, J. van der Zee, W. L. J. van Putten, G. C. van Rhoon, J. D. P. van Dijk, D. G. Gonzalez, F. F. Liu, P. Goodman, and M. Sherar, "Radiotherapy with or without hyperthermia in the treatment of superficial localized breast cancer: Results from five randomized controlled trials," Int. J. Rad. Oncol. Biol. Phys., vol. 35, no. 4, pp. 731-744, July 1996.
[2] J. W. Hand, D. Machin, C. C. Vernon, and J. B. Whaley, "Analysis of thermal parameters obtained during phase III trials of hyperthermia as an adjunct to radiotherapy in the treatment of breast carcinoma," Int. J. Hyperthermia, vol. 13, pp. 343-364, 1997.

[3] D. S. Kapp, "Efficacy of adjuvant hyperthermia in the treatment of superficial recurrent breast cancer: Confirmation and future directions," Int. J. Rad. Oncol. Biol. Phys., vol. 35, pp. 1117-1121, 1996.

[4] K. A. Leopold, M. Dewhirst, T. Samulski, J. Harrelson, J. A. Tucker, S. L. George, R. K. Dodge, W. Grant, S. Clegg, L. R. Prosnitz, and J. R. Oleson, "Relationships among tumor temperature, treatment time, and histopathological outcome using preoperative hyperthermia with radiation in soft-tissue sarcomas," Int. J. Rad. Oncol. Biol. Phys., vol. 22, no. 5, pp. 989-998, 1992.

[5] J. van der Zee, D. G. Gonzalez, G. C. van Rhoon, J. D. P. van Dijk, W. L. J. van Putten, and A. A. M. Hart, "Comparison of radiotherapy alone with radiotherapy plus hyperthermia in locally advanced pelvic tumors: A prospective, randomised, multicentre trial," Lancet, vol. 355, pp. 1119-1125, 2000.

[6] P. Wust, B. Hildebrandt, G. Sreenivasa, B. Rau, J. Gellermann, H. Riess, R. Felix, and P. M. Schlag, "Hyperthermia in combined treatment of cancer," Lancet Oncol., vol. 3, no. 8, pp. 487-497, Aug. 2002.

[7] J. Overgaard, D. Gonzalez, M. Hulshof, G. Arcangeli, O. Dahl, O. Mella, and S. Bentzen, "Randomized trial of hyperthermia as an adjuvant to radiotherapy for recurrent or metastatic malignant melanoma," Lancet, vol. 345 , pp. $540-543,1995$.

[8] R. B. Roemer, "Engineering aspects of hyperthermia therapy," in Annual Review of Biomedical Engineering, M. L. Yarmush, K. R. Diller, and M. Toner, Eds. Palo Alto, CA: Ann. Rev., 1999, vol. 1, pp. 347-376.

[9] C. J. Diederich and K. Hynynen, "Ultrasound technology for hyperthermia," Ultrasound Med. Biol., vol. 25, no. 6, pp. 871-887, 1999.

[10] H. Wan, P. VanBaren, E. S. Ebbini, and C. A. Cain, "Ultrasound surgery: Comparison of strategies using phased array systems," IEEE Trans. Ultrason., Ferroelect., Freq. Contr., vol. 43, pp. 1085-1098, Nov. 1996.

[11] V. Sathiaseelan, B. B. Mittal, A. J. Fenn, and A. Taflove, "Recent advances in external electromagnetic hyperthermia," in Advances in Radiation Therapy, B. B. Mittal, J. A. Purdy, and K. K. Ang, Eds. Boston, MA: Kluwer, 1998, ch. 10, pp. 213-245.

[12] M. E. Kowalski, B. Behnia, A. G. Webb, and J.-M. Jin, "Optimization of electromagnetic phased-arrays for hyperthermia via magnetic resonance temperature estimation," IEEE Trans. Biomed. Eng., vol. 49, pp. 1229-1241, Nov. 2002.

[13] M. E. Kowalski and J.-M. Jin, "A temperature-based feedback control system for electromagnetic phased-array hyperthermia: Theory and simulation," Phys. Med. Biol., vol. 48, pp. 633-651, 2003.

[14] A. J. Fenn, V. Sathiaseelan, G. A. King, and P. R. Stauffer, "Improved localization of energy deposition in adaptive phase-array hyperthermia treatment of cancer," Lincoln Lab. J., vol. 9, no. 2, pp. 187-195, 1996.

[15] A. J. Fenn, G. L. Wolf, and R. M. Fogle, "An adaptive microwave phase array for targeted heating of tumours in intact breast: Animal study results," Int. J. Hyperthermia, vol. 15, no. 1, pp. 45-61, 1999.

[16] S. Jacobsen, "Reduction of hot spots in hyperthermia by means of broadband energy transmission," Electron. Lett., vol. 34, no. 20, pp. 1901-1902, Oct. 1998.

[17] E. J. Bond, X. Li, S. C. Hagness, and B. D. Van Veen, "Microwave imaging via space-time beamforming for early detection of breast cancer," IEEE Trans. Antennas Propagat., vol. 51, pp. 1690-1705, Aug. 2003.

[18] S. Jacobsen and P. Stauffer, "Non-invasive temperature profile estimation in a lossy medium based on multi-band radiometric signals sensed by a microwave dual-purpose body-contacting antenna," Int. J. Hyperthermia, vol. 18, no. 2, pp. 86-103, 2002.

[19] S. Jacobsen and P. R. Stauffer, "Nonparametric 1-D temperature restoration in lossy media using Tikhonov regularization on sparse radiometry data," IEEE Trans. Biomed. Eng., vol. 50, pp. 178-188, Feb. 2003.

[20] P. Meaney, K. D. Paulsen, M. W. Fanning, D. Li, and Q. Fang, "Image accuracy improvements in microwave tomographic thermometry: Phantom experience," Int. J. Hyperthermia, vol. 19, no. 5, pp. 534-550, 2003.

[21] R. M. Arthur, W. L. Straube, J. D. Starman, and E. G. Moros, "Noninvasive temperature estimation based on the energy of backscattered ultrasound," Med. Phys., vol. 30, no. 6, pp. 1021-1029, June 2003.

[22] B. Van Veen and K. Buckley, "Beamforming: A versatile approach to spatial filtering," in IEEE Acoust. Speech, Signal Processing Mag., vol. 5, Apr. 1988, pp. 4-24.

[23] S. K. Davis, E. J. Bond, X. Li, S. C. Hagness, and B. D. Van Veen, "Microwave imaging via space-time beamforming for early detection of breast cancer: Beamformer design in the frequency domain," J. Electomagn. Waves Applicat., vol. 17, no. 2, pp. 357-381, 2003. 
[24] H. Cox, "Resolving power and sensitivity to mismatch of optimum array processors," J. Acoust. Soc. Amer, vol. 54, no. 3, pp. 771-785, 1973.

[25] H. Cox, R. M. Zeskind, and M. M. Owen, "Robust adaptive beamforming," in IEEE Trans. Acoust. Speech, Signal Processing, vol. ASSP-35, Oct. 1987, pp. 1365-1375.

[26] S. K. Davis, X. Li, E. J. Bond, S. C. Hagness, and B. D. Van Veen, "Frequency-domain penalized least-squares beamformer design for early detection of breast cancer via microwave imaging," in IEEE Sensor Array and Multichannel Signal Processing Workshop, Rosslyn, VA, Aug. 2002, pp. 120-124.

[27] X. Li, S. K. Davis, S. C. Hagness, D. W. van der Weide, and B. D. Van Veen, "Microwave imaging via space-time beamforming: Experimental investigation of tumor detection in multilayer breast phantoms," IEEE Trans. Microwave Theory Tech., vol. 52, pp. 1856-1865, Aug. 2004.

[28] X. Li and S. C. Hagness, "A confocal microwave imaging algorithm for breast cancer detection," IEEE Microwave Wireless Comp. Lett., vol. 11, pp. 130-132, Mar. 2001.

[29] O. P. Gandhi and C. M. Furse, "Currents induced in the human body for exposure to ultrawideband electromagnetic pulses," IEEE Trans. Electromagn. Compat., vol. 39, pp. 174-180, May 1997.

[30] J. Z. Bao, M. L. Swicord, and C. C. Davis, "Microwave dielectric characterization of binary mixtures of water, methanol, and ethanol," J. Chem. Phys., vol. 104, no. 12, pp. 4441-4450, Mar. 1996.

[31] P. Bernardi, M. Cavagnaro, S. Pisa, and E. Piuzzi, "Specific absorption rate and temperature elevation in a subject exposed in the far-field of radio-frequency sources operating in the 10-900-MHz range," IEEE Trans. Biomed. Eng., vol. 50, pp. 295-304, Mar. 2003.

[32] K. Sekins and A. Emery, "Thermal modeling of local hyperthermia," in Hyperthermia in Cancer Treatment. Boca Raton, FL: CRC, 1986, vol. 2 , ch. 6.

[33] H. Bowmann, "Heat transfer and thermal dosimetry," J. Microwave Power Elect. Eng., vol. 16, no. 2, pp. 121-130, 1981.

[34] J. Valvano, "Tissue thermal properties and perfusion," in $O p$ tical-Thermal Response of Laser-Irradiated Tissue. New York: Plenum, 1995, ch. 14

[35] M. G. Skinner, M. N. Iizuka, M. C. Kolios, and M. D. Sherar, "A theoretical comparison of energy sources-microwave, ultrasound and laser-for interstitial thermal therapy," Phys. Med. Biol., vol. 43, pp. $3535-3547,1998$

[36] K. Giering, I. Lamprecht, and O. Minet, "Specific heat capacities of human and animal tissues," Proc. SPIE, vol. 2624, pp. 188-197, Jan. 1996.

[37] J. C. Kumaradas and M. D. Sherar, "Optimization of a beam shaping bolus for superficial microwave hyperthermia waveguide applicators using a finite element method," Phys. Med. Biol., vol. 48, no. 1, pp. 1-18, Jan. 2003.

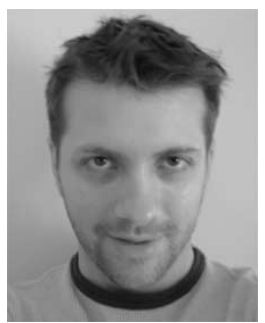

Mark Converse (S'92-M'04) received the B.S. degree in electrical engineering and M.S. and Ph.D. degrees from the University of Wisconsin-Madison, in 1996, 1999, and 2003, respectively.

During his graduate studies, he was engaged in plasma processing research involving damage evaluation/analysis and mitigation during the etching process. Upon receiving the M.S. degree in 1999, he began research in microwave vacuum electronics investigating the impulse response of the helix traveling wave tube. He is currently a Post-Doctoral Scholar with the University of Wisconsin-Madison, where he is involved with projects involving the microwave detection and treatment of breast and liver cancer. His research interests include EM interactions with materials, electrical/biological interfaces, and organic electronics.

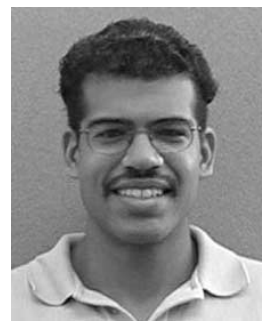

Essex J. Bond (S'98) was born in Milwaukee, WI. He received the B.S. degree from the Milwaukee School of Engineering, Milwaukee, WI, in 1999, the M.S. degree from the University of Wisconsin-Madison, in 2001, both in electrical engineering, and is currently working toward the $\mathrm{Ph} . \mathrm{D}$. degree in the electrical and computer engineering at the University of Wisconsin-Madison.

His research interests include statistical signal processing, biomedical applications of signal processing, adaptive filtering, and image-reconstruction

techniques.

Mr. Bond was the recipient of a National Institutes of Health Pre-Doctoral Fellowship

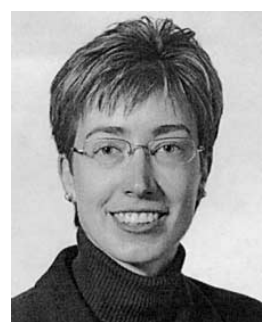

Susan C. Hagness (S'91-M'98) received the B.S. (with highest honors) and Ph.D. degree in electrical engineering from Northwestern University, Evanston, IL, in 1993 and 1998, respectively. While working toward the Ph.D. degree, she was a National Science Foundation (NSF) Graduate Fellow and a Tau Beta Pi Spencer Fellow.

Since August 1998, she has been with the University of Wisconsin-Madison, where she is currently an Associate Professor with the Department of Electrical and Computer Engineering and a faculty affiliate with the Department of Biomedical Engineering. Her research interests include FDTD theory and applications, microwave imaging and sensing techniques for biological and medical applications, and full-wave analysis and design of photonic microstructures. She coauthored Computational Electrodynamics: The Finite-Difference Time-Domain Method. (Boston, MA: Artech House, 2000, 2nd ed.).

Dr. Hagness is a member of the IEEE Antennas and Propagation Society (IEEE AP-S) Administrative Committee (AdCom). She is currently an associate editor for the IEEE ANTENNAS AND WIRELESS PROPAGATION LETTERS. She was an invited participant in the National Academy of Engineering's Frontiers of Engineering Symposium in 2002. She was the recipient of the Presidential Early Career Award for Scientists and Engineers presented by the White House in 2000. She was the recipient of the Booker Fellowship Award presented by the United States National Committee of the International Union of Radio Science in 2002. She was also named one of the 100 top young innovators in science and engineering in the world by the Massachusetts Institute of Technology (MIT) Technology Review magazine. In 2003, she was the recipient of the University of Wisconsin Emil Steiger Distinguished Teaching Award.

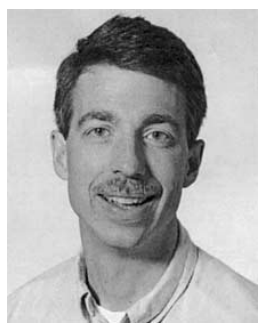

Barry D. Van Veen (S'81-M'86-SM'97-F'02) was born in Green Bay, WI. He received the B.S degree from the Michigan Technological University, Houghton, in 1983, and the Ph.D. degree from the University of Colorado at Boulder, in 1986, both in electrical engineering. He was an Office of Naval Research (ONR) Fellow while working toward the Ph.D. degree.

In Spring 1987, he was with the Department of Electrical and Computer Engineering, University of Colorado at Boulder. Since August 1987, he has been with the Department of Electrical and Computer Engineering, University of Wisconsin-Madison, where he is currently a Professor. He coauthored Signals and Systems (New York: Wiley, 1999, 1st ed.; 2003, 2nd ed.). His research interests include signal processing for sensor arrays, wireless communications, and biomedical applications of signal processing.

Dr. Van Veen was an associate editor for the IEEE TRANSACTIONS ON Signal Processing and the IEEE Signal Processing Society's Statistical Signal and Array Processing Technical Committee and the Sensor Array and Multichannel Technical Committee. He was a recipient of a 1989 Presidential Young Investigator Award presented by the National Science Foundation and a 1990 IEEE Signal Processing Society Paper Award. He was also the recipient of the Holdridge Teaching Excellence Award presented by the Department of Electrical and Computer Engineering, University of Wisconsin-Madison, in 1997. 